University of Nebraska - Lincoln

DigitalCommons@University of Nebraska - Lincoln

Publications from USDA-ARS / UNL Faculty

U.S. Department of Agriculture: Agricultural

Research Service, Lincoln, Nebraska

2006

\title{
RNA polymerase II gene (RPB2) encoding the second largest protein subunit in Phaeosphaeria nodorum and P. avenaria
}

\author{
Arkadiusz Malkus \\ Plant Breeding and Acclimatization Institute \\ Pi-Fang Linda Chang \\ National Chung Hsing University \\ Sabina M. Zuzga \\ Warsaw Agricultural University \\ Kuang-ren Chung \\ University of Florida \\ Jonathan Shao \\ Plant Science Institute \\ See next page for additional authors
}

Follow this and additional works at: https://digitalcommons.unl.edu/usdaarsfacpub

Part of the Agricultural Science Commons

Malkus, Arkadiusz; Chang, Pi-Fang Linda; Zuzga, Sabina M.; Chung, Kuang-ren; Shao, Jonathan; Cunfer, Barry M.; Arseniuk, Edward; and Ueng, Peter P., "RNA polymerase II gene (RPB2) encoding the second largest protein subunit in Phaeosphaeria nodorum and P. avenaria" (2006). Publications from USDA-ARS / UNL Faculty. 383.

https://digitalcommons.unl.edu/usdaarsfacpub/383

This Article is brought to you for free and open access by the U.S. Department of Agriculture: Agricultural Research Service, Lincoln, Nebraska at DigitalCommons@University of Nebraska - Lincoln. It has been accepted for inclusion in Publications from USDA-ARS / UNL Faculty by an authorized administrator of DigitalCommons@University of Nebraska - Lincoln. 


\section{Authors}

Arkadiusz Malkus, Pi-Fang Linda Chang, Sabina M. Zuzga, Kuang-ren Chung, Jonathan Shao, Barry M. Cunfer, Edward Arseniuk, and Peter P. Ueng 


\title{
RNA polymerase II gene (RPB2) encoding the second largest protein subunit in Phaeosphaeria nodorum and P. avenaria
}

\author{
Arkadiusz MALKUS ${ }^{a}$, Pi-Fang Linda CHANG $^{b}$, Sabina M. ZUZGA', \\ Kuang-ren $\mathrm{CHUNG}^{d}$, Jonathan SHAO ${ }^{e}$, Barry M. CUNFER, \\ Edward ARSENIUK ${ }^{a}$, Peter P. UENG ${ }^{e, *}$ \\ ${ }^{a}$ Department of Plant Pathology, Plant Breeding and Acclimatization Institute, Radzikow, Poland \\ ${ }^{b}$ Department of Plant Pathology, National Chung Hsing University, Taichung 402, Taiwan \\ ${ }^{\mathrm{c} D e p a r t m e n t}$ of Plant Genetics, Breeding and Biotechnology, Warsaw Agricultural University, Warsaw, Poland \\ ${ }^{\mathrm{d} C i t r u s ~ R e s e a r c h ~ \& ~ E d u c a t i o n ~ C e n t e r, ~ U n i v e r s i t y ~ o f ~ F l o r i d a, ~ L a k e ~ A l f r e d, ~ F L ~ 33850, ~ U S A ~}$ \\ ${ }^{\mathrm{e}}$ Molecular Plant Pathology Laboratory, Plant Science Institute, U.S. Department of Agriculture, ARS, \\ Bldg 004, Rm 118, 10300 Baltimore Ave., Beltsuille, MD 20705, USA \\ f Department of Plant Pathology, University of Georgia, Griffin, GA 30223, USA
}

\section{A R T I C L E I N F O}

Article history:

Received 8 June 2006

Received in revised form

7 July 2006

Accepted 28 July 2006

Published online 3 October 2006

Corresponding Editor: Paul Hooley

\section{Keywords:}

Cochliobolus heterostrophus

Enzymes

Plant pathology

Stagnospora nodorum

Stagnospora leaf blotch

\begin{abstract}
A B S T R A C T
A 5586 bp sequence (accession no. DQ278491), which includes the RNA polymerase II gene (RPB2) encoding the second largest protein subunit (RPB2), was obtained from the wheat biotype Phaeosphaeria nodorum (PN-w) by PCR amplification. The $3841 \mathrm{bp}$ full length RPB2 gene contains two exons and a 52 bp intron, and encodes a complete 1262 amino acid protein. Similar to the C-terminals of the $\beta$ subunits of prokaryotes and yeast RNA polymerases, the deduced RPB2 protein contained many structural features needed for gene transcription. Based on the phylogenetic analysis with the deduced RPB2 polypeptide sequences, the $\mathrm{PN}-\mathrm{w}$ was closely related to the maize pathogen Cochliobolus heterostrophus. Size differences were found in the full length RPB2 gene of cereal Phaeosphaeria species, mainly due to differences in intron size. No nucleotide substitutions were found in homothallic P. avenaria f.sp. triticea (Pat1) and barley biotype P. nodorum (PN-b) isolates used in this study. The nucleotide and deduced amino acid sequences of the RPB2 gene in Pat1 were closely related to that in $\mathrm{PN}-\mathrm{w}$.

(c) 2006 The British Mycological Society. Published by Elsevier Ltd. All rights reserved.
\end{abstract}

\section{Introduction}

Phaeosphaeria nodorum (syn. Leptosphaeria nodorum; anamorph: Stagonospora nodorum, Syn. Septoria nodorum) and Phaeosphaeria avenaria (Syn. Leptosphaeria avenaria; anamorph: Stagonospora avenae, Syn. Septoria avenae), are two of the most important causal agents of cereal Stagonospora leaf blotch diseases (Couture 1989; Noble \& Montgomerie 1954; Scharen \&
Sanderson 1985; Sprague 1950; Weber 1922a,b). The identification of these two pathogens is based largely on morphology of the anamorph (Cunfer 1997, 2000; Cunfer \& Ueng 1999; Richardson \& Noble 1970). Based on its pathogenicity to wheat (Triticum aestivum) or barley (Hordeum vulgare), P. nodorum was further recognized as having two special forms, a wheat biotype (PN-w) and a barley biotype (PN-b) (Holmes \& Colhoun 1970; Smedegård-Petersen 1974). P. avenaria was also divided

\footnotetext{
* Corresponding author. Tel.: +1 301504 6308; fax: +1 3015045449.

E-mail address: uengp@ba.ars.usda.gov.

0953-7562/\$ - see front matter ๔ 2006 The British Mycological Society. Published by Elsevier Ltd. All rights reserved.
}

doi:10.1016/j.mycres.2006.07.015 
into two groups; P. avenaria f.sp. avenaria (Paa) which infects oats (Avena spp.), and P. avenaria f.sp. triticea (Pat) which infects wheat, barley, rye (Secale cereale) and several grasses (Johnson 1947; Shaw 1957).

In recent years, genetic relatedness and differentiation of cereal Phaeosphaeria species have been examined at the molecular level. Sequence data from rDNA ITS, mating type gene (mat1) conserved regions, and genes encoding glyceraldehyde-3-phosphate dehydrogenase (gpd), $\beta$-glucosidase (bgl1) and $\beta$-tubulin (tubA) have been used to define phylogenetic relationships among $\mathrm{PN}-\mathrm{w}$ and $\mathrm{PN}-\mathrm{b}$, three genetically distinct groups of Pat (homothallic Pat1, heterothallic Pat2 and Pat3), and Paa (Malkus et al. 2005; Reszka et al. 2005; Ueng et al. 1998, 2003a,b). In 1995, based on sequence diversity, a new Phaeosphaeria sp. isolated from Polish winter rye was identified (Reszka et al. 2006). This new species has similar asexual pycnidiospore morphology and causes the same disease symptoms in cereals as P. nodorum. However, this new species has different intron sizes and sequences in the bgl1 and tubA genes (Malkus et al. 2005; Reszka et al. 2005).

In addition to ITS and LSU and SSU rDNA, sequence data from genes encoding structural and functional proteins have recently been used to broaden the base of molecular characters that play important roles in phylogenetic studies of eukaryotes. It was suggested that the single-copy genes, which encode polypeptides with sufficient length ( 400 amino acids), and $\geq 65-70 \%$ identities among all taxa are good candidates for resolving the deep phylogenetic relations in fungi (Baldauf et al. 2000; Sicheritz-Pontén \& Andersson 2001). RNA polymerase II is one of the three RNA polymerases in eukaryotes which catalyse the transcription of messenger RNA. In yeasts, RNA polymerase II holoenzyme is composed of ten to twelve protein subunits (RPB1-RPB12), and the genes encoding these protein subunits are scattered throughout the genome (Archambault \& Friesen 1993; Ishihama et al. 1998; Sakurai \& Ishihama 1997; Young 1991). The RNA polymerase II gene (RPB2), which encodes the second largest protein subunit, is suggested to be a single-copy gene in fungi. The second largest protein subunit of RNA polymerase II (RPB2) is well conserved. Twelve highly conserved regions of the RPB2 protein subunit have $>85 \%$ amino acid identity among fungi, plants, and animals (James et al. 1991). Therefore, in combination with numerous morphological characteristics and other nucleotide and deduced polypeptide sequence data, the partial RPB2 gene sequence has been used in parsimony analysis to detect phylogenetic links and to understand evolution in higher ascomycetes and lichen-forming lower ascomycetes (Chaverri \& Samuels 2003; Chaverri et al. 2003; Hansen et al. 2005; Liu \& Hall 2004; Liu et al. 1999; Lumbsch 2000; Lutzoni et al. 2004; Miller \& Huhndorf 2005; Reeb et al. 2004; Staats et al. 2005; Zhang \& Blackwell 2001, 2002; Zhong \& Pfister 2004).

Based on the sequences of ITS, mat1 conserved regions and the bgl1, gpd and tubA genes, the phylogenetic relationships of Phaeosphaeria species have been discussed (Malkus et al. 2005; Reszka et al. 2005; Ueng et al. 1998, 2003a,b). Whether the RPB2 gene and its deduced amino acid sequences could be used to support the phylogenetic relationships of cereal Phaeosphaeria species remained to be studied. Developing a reliable molecular method for Phaeosphaeria identification will not only help to diagnose cereal Stagonospora leaf blotch diseases, but also help to develop potential disease control strategies. Hence, in this study, the sequence variation of the RPB2 gene was used to develop a rapid assay for these two important cereal Phaeosphaeria species. In addition, the RPB2 gene may assist in the detection and differentiation of other grass Phaeosphaeria species.

\section{Materials and methods}

The RPB2 gene from wheat biotype $P$. nodorum was amplified from the isolate Sn37-1. Procedures for fungal culture in a liquid medium and for genomic DNA (gDNA) isolation were described previously (Ueng et al. 1992). The partial RPB2 gene was first PCR-amplified with degenerate primer sets, fRPB2-5F/fRPB2-7cR and $f R P B 2-7 c F / R P B 2-11 b R$, which represent three separate amino acid-conserved motifs (5th, 7th and 11th) in the RPB2 polypeptide throughout eukaryotes (Figs 1-2, Table 1) (Liu et al. 1999). These two DNA sequences were joined by a fragment amplified with the primer set 2A/

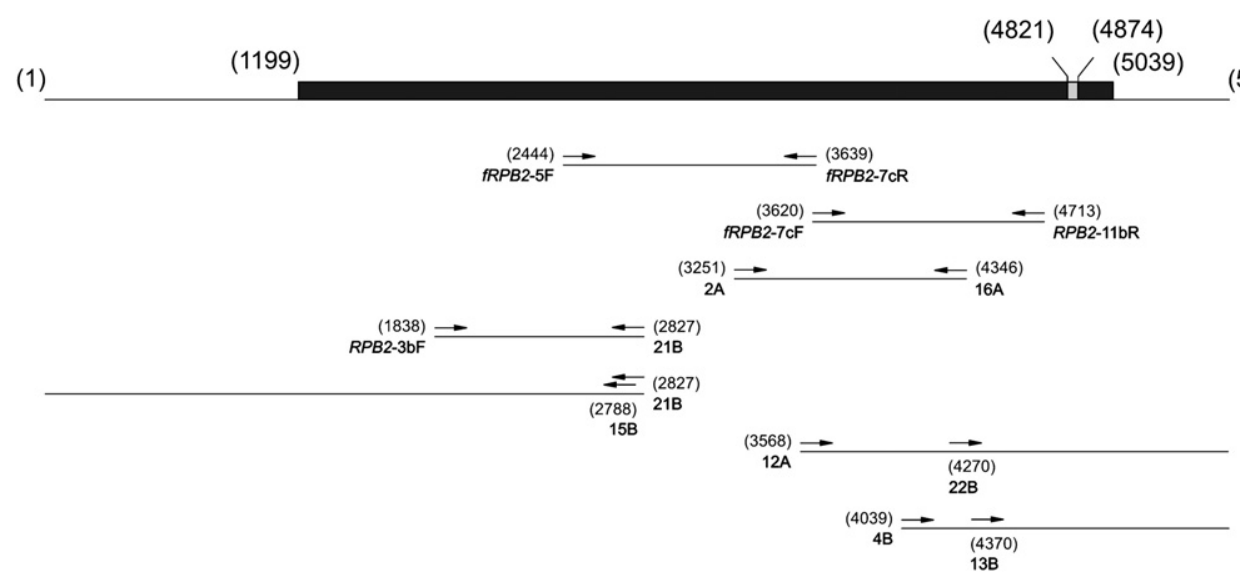

(5586)

\footnotetext{
Fig 1 - PCR strategies for isolation of the full-length RPB2 gene encoding the second largest subunit of RNA polymerase II in wheat biotype Phaeosphaeria nodorum. Two exons (nt1199-nt4821 and nt4874-nt5039) are shown in black boxes, and one intron (between nt4822 and nt4873) in grey box. The PCR primers with their amplifying directions are indicated in arrows, and nucleotide positions corresponding to the full-length sequence of the RPB2 gene are shown in parentheses.
} 


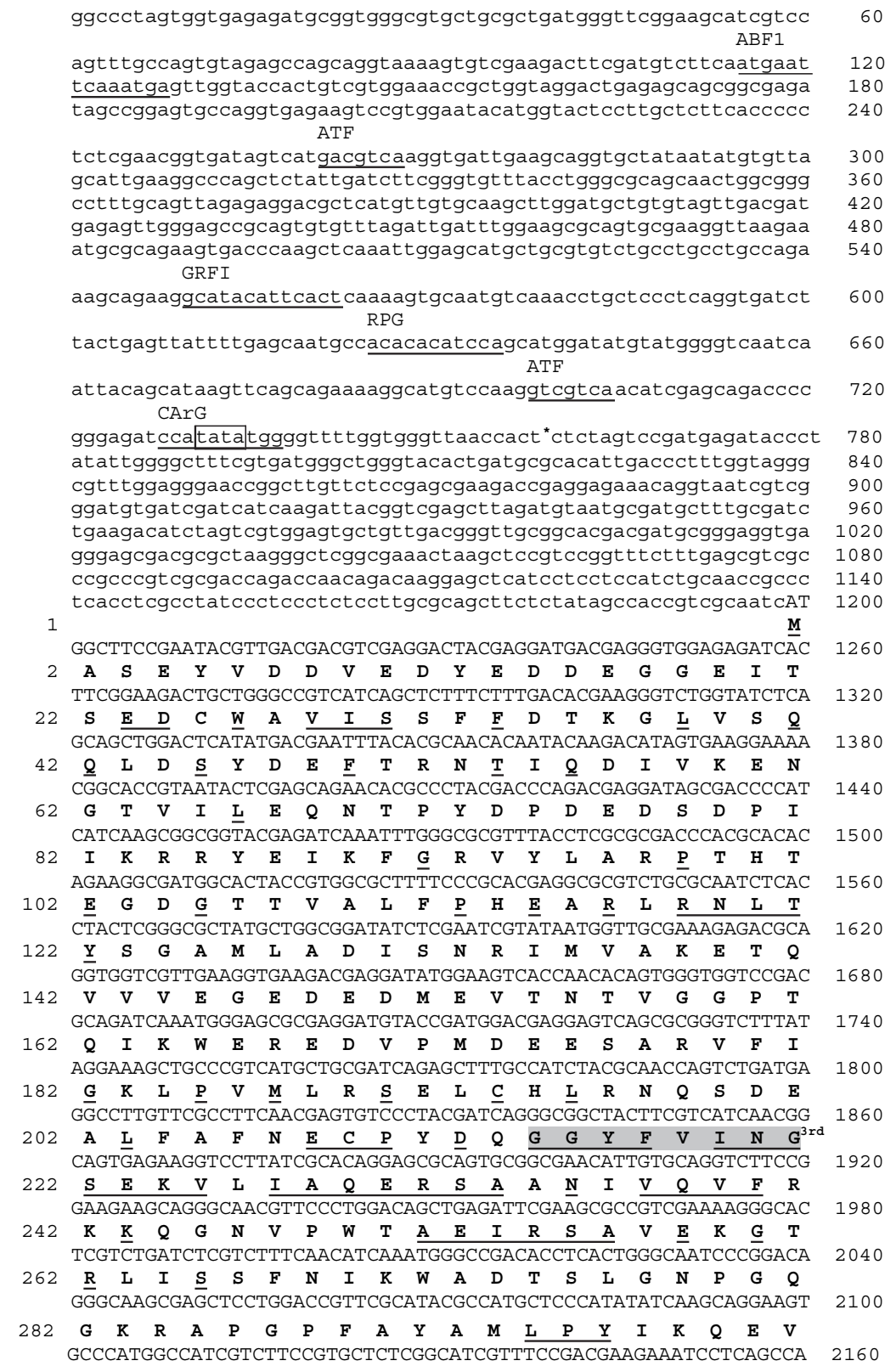

Fig 2 - Nucleotide and deduced peptide sequences of the RPB2 gene encoding the second largest subunit of RNA polymerase II in wheat biotype Phaeosphaeria nodorum isolate Sn37-1. The numbers in the right column are for nucleotide numbering, and the ones in the left column for amino acids. The transcription factor binding sites ABF1, ATF, CArG, GRFI and RPG are underlined. The putative TATA box is boxed. The asterisk (*) indicates the potential transcription starting site (TSS). Lowercase letters indicate the non-translated regions of the RPB2 gene including an intron. The doubleunderlined nucleotides denote the position of a putative polyadenylation signal. Letters in bold, uppercase and below the nucleotide coding sequence indicate the putative peptide sequence. Four amino acid motifs $\left(3^{\text {rd }}, 5^{\text {th }}, 7^{\text {th }}\right.$ and $\left.11^{\text {th }}\right)$ that are conserved throughout eukaryotes and were used for the design of degenerate primers for PCR amplification are shaded. Underlined amino acids are conserved in full-length RPB2 subunit of RNA polymerase II found in 11 ascomycetes. Period (.) is the stop codon. The open circles above amino acids (GX 5 GKT) (aa942-aa950) are the purine nucleotide-binding motif, and the solid circles $\left(\mathrm{CX}_{2} \mathrm{CX}_{15} \mathrm{CX}_{2} \mathrm{C}\right)$ (aa1198-aa1220) indicate the zinc-binding motif. Two amino acid sequence clusters (aa997-aa1014 and aa1129-aa1137) involved in the formation of the active site of RNA polymerase are over-lined, and two potential lysine amino acids $\left(\mathrm{K}_{1014}\right.$ and $\left.\mathrm{K}_{1137}\right)$ for nucleotide analogues affinity labelling within the active site are indicated by open squares. Solid triangles indicate the bacterial RNase conserved amino acids residues. 
$\begin{array}{lllllllllllllllllllll}302 & \text { P } & \text { M } & \text { A } & \text { I } & \text { V } & \text { F } & \text { R } & \text { A } & \text { L } & \text { G } & \text { I } & \text { V } & \text { S } & \text { D } & \text { E } & \text { E } & \text { I } & \text { L } & \text { S } & \underline{H}\end{array}$ CATCGTATACGACCGCACCGACACACAGATGCTGGAGTTACTGAAGCCTAGCATCGAGGA 2220

$\begin{array}{llllllllllllllllllllll}322 & \text { I } & \mathbf{V} & \text { Y } & \underline{D} & \text { R } & \text { T } & \underline{D} & \text { T } & \text { Q } & \underline{M} & \text { L } & \text { E } & \text { L } & \text { L } & \text { K } & \underline{P} & \text { S } & \text { I } & \underline{E} & \text { E }\end{array}$ GĞGTGCGGTGĀTACAGGACĀGGGAGACTGCACTCGACTTCATCGCCĀAGCGTGGĀ̄ CCAA 2280

$\begin{array}{lllllllllllllllllllllll}342 & \text { G } & \text { A } & \mathbf{V} & \text { I } & \text { Q } & \text { D } & \text { R } & \text { E } & \text { T } & \underline{\mathbf{A}} & \text { L } & \text { D } & \text { F } & \text { I } & \text { A } & \text { K } & \text { R } & \text { G } & \text { A } & \text { N }\end{array}$

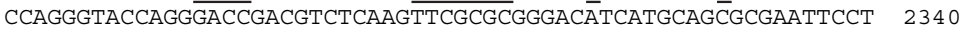

$\begin{array}{llllllllllllllllllllll}362 & Q & G & T & R & D & R & R & \text { L } & \text { K } & \text { F } & \text { A } & \text { R } & \text { D } & \text { I } & \text { M } & \text { Q } & \text { R } & \text { E } & \text { F } & \text { L }\end{array}$

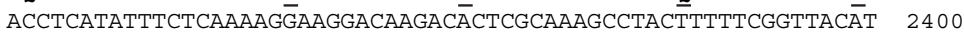

$\begin{array}{llllllllllllllllllllll}382 & \boldsymbol{P} & \text { H } & \text { I } & \text { S } & \text { Q } & \text { K } & \text { E } & \text { G } & \text { Q } & \text { D } & \text { T } & \text { R } & \text { K } & \text { A } & \text { Y } & \text { F } & \text { F } & \text { G } & \text { Y } & \text { M }\end{array}$ GATCCACCGACTGTTGCAGTGTGTTCTCGGGCGCCGAGACGAAGACGACCGA $\frac{\mathbf{T}}{2 \mathrm{GATTT}} 2460$

$\begin{array}{lllllllllllllllllllllll}402 & \text { I } & H & \text { R } & \text { L } & \text { L } & \text { Q } & \text { C } & \text { V } & \text { L } & \text { G } & \underline{R} & \text { R } & \text { D } & \text { E } & \text { D } & \text { D } & \text { R } & \text { D } & \text { H } & \text { F }\end{array}$

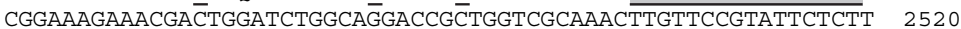

$\begin{array}{llllllllllllllllllll}422 & \mathbf{G}^{5 \mathrm{th}} \mathrm{K} & \mathbf{K} & \mathbf{R} & \mathbf{L} & \text { D } & \text { L } & \text { A } & \text { G } & \text { P } & \text { L } & \text { V } & \text { A } & \text { N } & \text { L } & \text { F } & \text { R } & \text { I } & \text { L } & \text { F }\end{array}$ CCTGAAGTTGACTAAGGACGTCTTCAAGTATCTTCAGCGATGCGTCGAGAGCAGCACGGA 2580

$\begin{array}{lllllllllllllllllllll}442 & \text { L } & \text { K } & \text { L } & \text { T } & \text { K } & \text { D } & \text { V } & \text { F } & \text { K } & \text { Y } & \text { L } & \text { Q } & \text { R } & \text { C } & \text { V } & \text { E } & \text { S } & \text { S } & \text { T } & \text { D }\end{array}$ CTTCAACGTGC̄AAATGḠCTGTGAAGGCCAGCATCATCACAAATGGCCTGAAATACTCTTT 2640

$\begin{array}{lllllllllllllllllllllll}462 & \text { F } & \text { N } & \text { V } & \text { Q } & \text { M } & \text { A } & \text { V } & \text { K } & \text { A } & \text { S } & \text { I } & \text { I } & \text { T } & \text { N } & \text { G } & \text { L } & \text { K } & \text { Y } & \text { S } & \text { L }\end{array}$

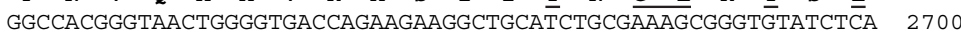

\begin{tabular}{lllllllllllllllllllll}
482 & $\mathbf{A}$ & $\mathbf{T}$ & $\mathbf{G}$ & $\mathbf{N}$ & $\mathbf{W}$ & $\mathbf{G}$ & $\mathbf{D}$ & $\mathbf{Q}$ & $\mathbf{K}$ & $\mathbf{K}$ & $\mathbf{A}$ & $\mathbf{A}$ & $\mathbf{S}$ & $\mathbf{A}$ & $\mathbf{K}$ & $\mathbf{A}$ & $\mathbf{G}$ & $\mathbf{V}$ & $\mathbf{S}$ & $\mathbf{Q}$ \\
\hline
\end{tabular} AGTGTTGAACCGCTACACATATGCATCTACATTGTCCCATTTGCGGAGGACGAACACGCC 2760

\begin{tabular}{llllllllllllllllllllll}
502 & V & L & N & R & Y & T & Y & A & S & T & L & S & H & L & R & R & T & N & T & P \\
\hline
\end{tabular} A $\overline{\text { GTCGGTCGTGACGGTAAGCTGGCGAAGCCTCGTCAGCTACACAACTCGCATTGGGGTCT }} 2820$

$\begin{array}{lllllllllllllllllllll}522 & \text { V } & \text { G } & \text { R } & \text { D } & \text { G } & \text { K } & \text { L } & \text { A } & \text { K } & \text { P } & \text { R } & \text { Q } & \text { L } & \text { H } & \text { N } & \text { S } & \text { H } & \text { W } & \text { G } & \text { L }\end{array}$ TGTTTGCCCTGCTGAAACCCCA AAAGGCCAGGCCTGTGGTCTGGTCAAGAACTTGTCCCT 2880

$\begin{array}{lllllllllllllllllllll}542 & \text { V } & \text { C } & \text { P } & \text { A } & \text { E } & \text { T } & \text { P } & \text { E } & \text { G } & \text { Q } & \text { A } & \text { C } & \text { G } & \text { L } & \text { V } & \text { K } & \text { N } & \text { L } & \text { S } & \text { L }\end{array}$ CATGTGCTACGTCAGTGTCGGTAGTGATGCTACCCCGATCATCGACTTCATGTCACAAĀG 2940

$\begin{array}{lllllllllllllllllllll}562 & \text { M } & \text { C } & \text { Y } & \text { V } & \text { S } & \text { V } & \text { G } & \text { S } & \text { D } & \text { A } & \text { T } & \text { P } & \text { I } & \text { I } & \text { D } & \text { F } & \text { M } & \text { S } & \mathbf{Q} & \mathbf{R}\end{array}$ AÄACATGCAACTCCTTGAGGAATACGACCAGAACCAAAACCCAGAAGCTACGAAGGTCTT 3000

$\begin{array}{lllllllllllllllllllll}582 & \mathbf{N} & \mathbf{M} & \boldsymbol{Q} & \mathrm{L} & \mathrm{L} & \mathbf{E} & \mathbf{E} & \mathbf{Y} & \mathrm{D} & \boldsymbol{Q} & \mathbf{N} & \boldsymbol{Q} & \mathbf{N} & \mathbf{P} & \mathbf{E} & \mathbf{A} & \mathbf{T} & \mathrm{K} & \mathrm{V} & \underline{\mathrm{F}}\end{array}$ CGTAAACGGTGTCTGGGTTGGTGTCCATTCTCAAGCGCAACAGCTCGTTTCTGTGGTTCA 3060

$\begin{array}{lllllllllllllllllllll}602 & \text { V } & \text { N } & \text { G } & \text { V } & \text { W } & \text { V } & \text { G } & \text { V } & \text { H } & \text { S } & \boldsymbol{Q} & \text { A } & \boldsymbol{Q} & \boldsymbol{Q} & \text { L } & \text { V } & \text { S } & \text { V } & \text { V } & \text { Q }\end{array}$ AGAG $\overline{C T G A}$ GAAGGĀACGGA $\bar{A} C T C T C T C G T A C G A A A T G A G C T T G \overline{T T C G T G A T A T C C G T G A} \quad 3120$

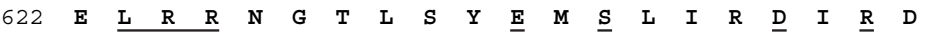
CCGGGAATTCAAGATCTTCACTGACGCTGGGCTTGTCĀTGCGACCTCTCTTCGTAGTAGA 3180

$\begin{array}{lllllllllllllllllllll}642 & \mathbf{R} & \mathbf{E} & \mathbf{F} & \mathrm{K} & \mathbf{I} & \mathbf{F} & \mathbf{T} & \mathbf{D} & \mathbf{A} & \mathbf{G} & \mathbf{R} & \mathbf{V} & \mathbf{M} & \mathbf{R} & \mathbf{P} & \mathbf{L} & \mathbf{F} & \mathrm{V} & \mathrm{V} & \mathbf{E}\end{array}$

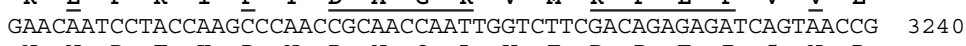

$\begin{array}{llllllllllllllllllllll}662 & \mathbf{N} & \mathbf{N} & \mathbf{P} & \mathbf{T} & \mathrm{K} & \mathbf{P} & \mathbf{N} & \mathbf{R} & \mathbf{N} & \mathbf{Q} & \mathbf{L} & \mathbf{V} & \mathbf{F} & \mathbf{D} & \mathbf{R} & \mathbf{E} & \mathbf{I} & \mathbf{S} & \mathbf{N} & \mathbf{R}\end{array}$ CCTTGTAAAGGAGCAGTTGGATAGCGACACTCGCGCCGGCTGGAGTGACGCTGACATCGC 3300

$\begin{array}{lllllllllllllllllllll}682 & \text { L } & \text { V } & \text { K } & \text { E } & \text { Q } & \text { L } & \text { D } & \text { S } & \text { D } & \text { T } & \text { R } & \text { A } & \text { G } & \text { W } & \text { S } & \text { D } & \text { A } & \text { D } & \text { I } & \text { A }\end{array}$ TGACAATACCTATGGATGGAAGGGACTCATCCAAGACGGTGTGATCGAGTACCTTGATGC 3360

$\begin{array}{lllllllllllllllllllll}702 & D & N & T & Y & G & \text { W } & \text { K } & \text { G } & \text { L } & \text { I } & \text { Q } & \text { D } & \text { G } & \text { V } & \text { I } & \text { E } & \text { Y } & \text { L } & \text { D } & \text { A }\end{array}$

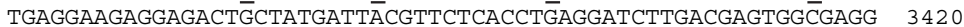

$\begin{array}{lllllllllllllllllllll}722 & \mathbf{E} & \mathbf{E} & \mathbf{E} & \mathbf{E} & \mathbf{T} & \mathbf{A} & \mathbf{M} & \mathbf{I} & \mathbf{T} & \mathbf{F} & \mathbf{S} & \mathbf{P} & \mathbf{E} & \mathbf{D} & \mathbf{L} & \mathbf{D} & \mathbf{E} & \mathbf{W} & \mathbf{R} & \mathbf{G}\end{array}$ CĀTGAAGATGGGTCTCCCAACGĀGCGAGCGAGCCAATCTTGGCAAGGAGCGTCTTCGACG 3480

$\begin{array}{llllllllllllllllllllll}742 & \mathbf{M} & \mathbf{K} & \mathbf{M} & \mathbf{G} & \text { L } & \mathbf{P} & \mathbf{T} & \mathbf{S} & \mathbf{E} & \mathbf{R} & \mathbf{A} & \mathbf{N} & \mathbf{L} & \mathbf{G} & \mathbf{K} & \mathbf{E} & \mathbf{R} & \mathbf{L} & \mathbf{R} & \mathbf{R}\end{array}$ TATCAAGCCCAAGCCGGACCCTCGCATCCACGCCTACACACATTGCGAGATTCATCCTG $\bar{C} \quad 3540$

$\begin{array}{lllllllllllllllllllll}762 & \text { I } & \text { K } & \text { P } & \text { K } & \text { P } & \text { D } & \text { P } & \text { R } & \text { I } & \text { H } & \text { A } & \text { Y } & \text { T } & \text { H } & \text { C } & \text { E } & \text { I } & \text { H } & \text { P } & \text { A }\end{array}$ TATGATTCTGGGTATATGCGCCAGTATCATTCCGTTCCCTGACCACAATCAGTCGCCCCG 3600

$\begin{array}{llllllllllllllllllllll}782 & \text { M } & \text { I } & \text { L } & \text { G } & \text { I } & \text { C } & \text { A } & \text { S } & \text { I } & \text { I } & \text { P } & \text { F } & \text { P } & \text { D } & \text { H } & \text { N } & \text { Q } & \text { S } & \text { P } & \text { R }\end{array}$ TÄACACATACCAGTCTGCCATGGGTAAGAAGCCATGGGTGTTGCCCTGACCAACTATGC 3660

$\begin{array}{lllllllllllllllllllllll}802 & \mathbf{N} & \mathbf{T} & \mathbf{Y} & \mathbf{Q} & \mathbf{S} & \mathbf{A} & \mathbf{M} & \mathbf{G} & \mathbf{K} & \mathbf{Q} & \mathbf{A} & \mathbf{M} & \mathbf{G}^{7 \text { th }} \mathrm{V} & \mathbf{A} & \mathbf{L} & \mathbf{T} & \mathbf{N} & \mathbf{Y} & \mathbf{A}\end{array}$ TCTGCGTATGGAGACGATGATGAACGTCCTTTATTATCCGCAAAAGCCTCTGGCCACAAC 3720

$\begin{array}{lllllllllllllllllllll}822 & \mathbf{L} & \mathbf{R} & \mathbf{M} & \mathbf{E} & \mathbf{T} & \mathbf{M} & \mathbf{M} & \mathbf{N} & \mathbf{V} & \mathbf{L} & \mathbf{Y} & \mathbf{Y} & \mathbf{P} & \mathbf{Q} & \mathbf{K} & \mathbf{P} & \mathbf{L} & \mathbf{A} & \mathbf{T} & \mathbf{T}\end{array}$ TCGCTCTATGGAGTACCTCAAGTTCCGTAAGCTGCCTGCTGGTCAAAACGCCATC $\overline{\text { GTCGC }} \quad 3780$

$\begin{array}{lllllllllllllllllllll}842 & \mathbf{R} & \mathbf{S} & \mathbf{M} & \mathbf{E} & \mathbf{Y} & \text { L } & \mathbf{K} & \mathbf{F} & \mathbf{R} & \mathbf{E} & \text { L } & \text { P } & \text { A } & \text { G } & \mathbf{Q} & \text { N } & \text { A } & \text { I } & \text { V } & \text { A }\end{array}$ CĀTCGCATGTTATTCTGGTTACAACCAAGAAGATTCCGTCATCATGAACCAAAGCAGTAT 3840

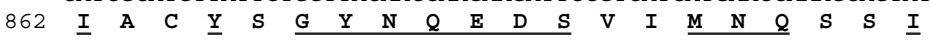

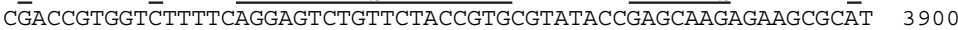

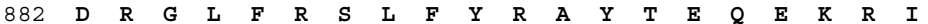

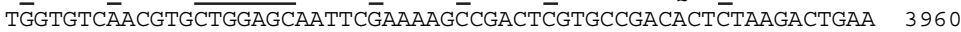

$\begin{array}{lllllllllllllllllllll}902 & G & V & \text { N } & \text { V } & \text { L } & \text { E } & \text { Q } & \text { F } & \text { E } & \text { K } & \underline{P} & \text { T } & \underline{R} & \text { A } & \text { D } & \text { T } & \text { L } & R & \text { L } & \text { K }\end{array}$ GG̈GCGGAACTTACGACÄAGCTCGACGACGATĞGTGTTGTCGCCCCCĞGAGTGCGTGTTTC 4020

$\begin{array}{lllllllllllllllllllll}922 & G & G & \text { T } & \text { Y } & \text { D } & \text { K } & \text { L } & \text { D } & \text { D } & \text { D } & \text { G } & \text { V } & \text { V } & \text { A } & \text { P } & \text { G } & \text { V } & \text { R } & \text { V } & \text { S }\end{array}$ TGGTGACGATATCATCATCGGAAAGACGGCGCAATAGCAGCTGATGCCCAA $\overline{\text { GAGCTGGG }} 4080$

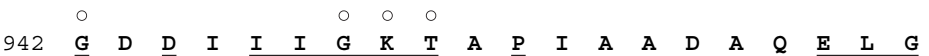
CСAGAAGĀCTACTCTACATACCAAGCGCGACGTGTCTACGCCTCTGCGAAGCACAGAGAA 4140

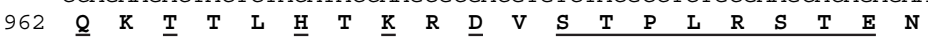

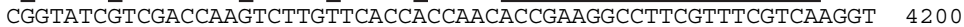

Fig 2 (continued)

$16 \mathrm{~A}$, and extended to the $5^{\prime}$ end of the RPB2 gene with the primer set RPB2-3bF/21B (Liu et al. 1999) (Figs 1-2, Table 1). PCR amplification was performed in $50 \mu$ reaction mixtures containing $1 \times$ reaction buffer $(50 \mathrm{~mm} \mathrm{KCl,} 10 \mathrm{~mm}$ Tris- $\mathrm{HCl}$,
$\mathrm{pH} 9.0$ at $25^{\circ} \mathrm{C}, 0.1 \%$ Triton $\left.\mathrm{X}-100\right), 1.25 \mathrm{~mm} \mathrm{MgCl}_{2}, 0.2 \mathrm{~mm}$ dNTPs, $2 \mu \mathrm{m}$ of each primer, $80 \mathrm{ng}$ gDNA, and 1.0 unit of Taq DNA polymerase (Promega, Madison, WI). Reaction parameters were: denaturation $\left(94^{\circ} \mathrm{C}, 3 \mathrm{~min}\right.$ ) followed by 40 cycles 


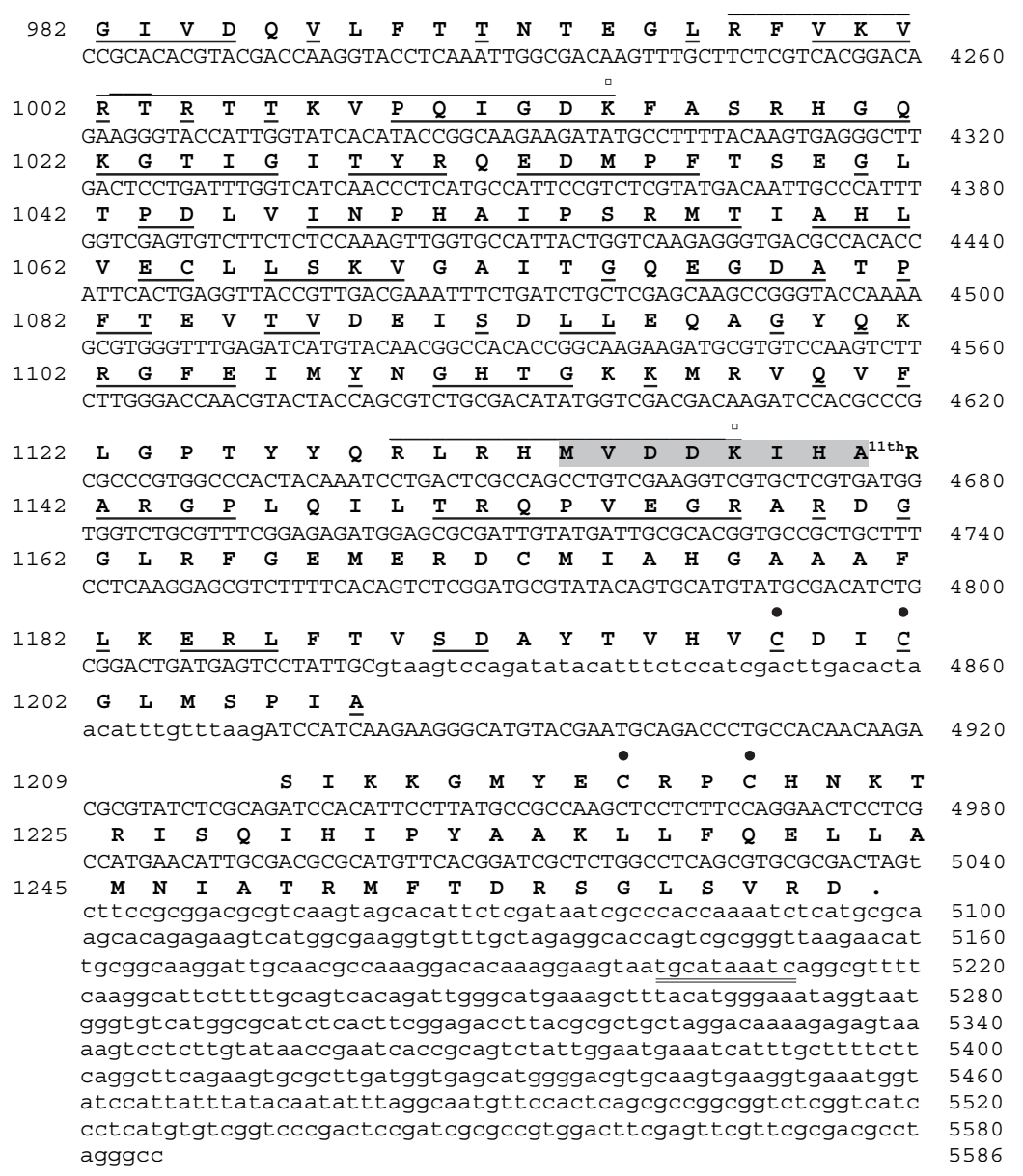

Fig 2 (continued)

of $94^{\circ} \mathrm{C}(20 \mathrm{~s}), 55^{\circ} \mathrm{C}(30 \mathrm{~s})$, and $72{ }^{\circ} \mathrm{C}(1 \mathrm{~min})$, and a final incubation at $72{ }^{\circ} \mathrm{C}(10 \mathrm{~min})$. In this way, a $2876 \mathrm{bp}$ partial RPB2 gene sequence was obtained.

The $5^{\prime}$ and $3^{\prime}$ flanking genomic RPB2 gene sequences were obtained with a 'step-down' PCR amplification technique (Zhang \& Gurr 2002). Endonuclease restrictions and adaptor ligations of gDNA followed the protocols as described (Zhang \& Gurr 2002). PCR amplification was performed in $20 \mu \mathrm{l}$ reaction mixtures containing $1 \times$ reaction buffer ( $15 \mathrm{~mm}$ potassium acetate, $40 \mathrm{~mm}$ tricine-potassium hydroxide, $\mathrm{pH} 9.2$ at $25^{\circ} \mathrm{C}, 3.5 \mathrm{~mm} \mathrm{Mg}$ acetate and bovine serum albumin $3.75 \mu \mathrm{g} \mathrm{ml}^{-1}$ ), $0.2 \mathrm{~mm}$ dNTPs, $10 \mu \mathrm{m}$ of each primer, $10 \mathrm{ng}$ ligated gDNA, and $1 \times$ Advantage cDNA polymerase mix (BD Biosciences-Clontech, Mountain View, CA). Reaction parameters were: three cycles of $94^{\circ} \mathrm{C}$ for $2 \mathrm{~s}$ and $72{ }^{\circ} \mathrm{C}$ for $3 \mathrm{~min}$, three cycles of $94^{\circ} \mathrm{C}$ for $2 \mathrm{~s}$ and $70{ }^{\circ} \mathrm{C}$ for $3 \mathrm{~min}$, and three cycles of $94^{\circ} \mathrm{C}$ for $2 \mathrm{~s}$ and $68^{\circ} \mathrm{C}$ for $3 \mathrm{~min}$, followed by 26 cycles of $94^{\circ} \mathrm{C}(2 \mathrm{~s}), 66^{\circ} \mathrm{C}(20 \mathrm{~s})$, and $68^{\circ} \mathrm{C}(3 \mathrm{~min})$, and a final incubation at $68^{\circ} \mathrm{C}(8 \mathrm{~min})$. Three DNA fragments representing the $5^{\prime}$ flanking region of the partial RPB2 gene were first amplified from SpeI, $\mathrm{XmaI}$ and BspEI enzyme restricted/adaptor ligated gDNA fragments with the primer set 21B/PP1, and subsequently with the nested primer set 15B/PP2 (Fig 1, Table 1). For the $3^{\prime}$ flanking region, three SpeI, NheI and Xbal enzyme restricted/adaptor ligated gDNA fragments were amplified with the primer set 12A/PP1 and then with the nested primer set 22B/PP2. The fourth PCR product was amplified from BspEI restricted/adaptor ligated gDNA fragments with the primer set 4B/PP1 and the nested one, 13B/PP2 (Fig 1, Table 1). Both primers PP1 and PP2 recognized sequences on the adaptors 1 and 2, and served as the upstream primers for PCR (Zhang \& Gurr 2002). Primers 21B, 15B, $12 \mathrm{~A}, 22 \mathrm{~B}, 4 \mathrm{~B}$ and $13 \mathrm{~B}$ were designed from the obtained 2876 bp partial RPB2 gene sequence as described above. Isolation and direct sequencing of PCR products were conducted as described previously (Ueng et al. 2003b).

To determine the expressed RPB2 gene sequence, total RNA was isolated from the wheat biotype $P$. nodorum isolate Sn37-1 culture grown in a liquid medium containing $0.5 \%$ malt extract, $0.5 \%$ yeast extract and $1 \%$ cellulose with shaking at $125 \mathrm{rev} \mathrm{min}^{-1}$ for $14 \mathrm{~d}$ at $27^{\circ} \mathrm{C}$. The mycelia were harvested, washed with $2 \%$ sodium chloride solution and flash-frozen in liquid nitrogen. The total RNA was extracted from mortar and pestle pulverized mycelia using the RNeasy Plant Mini Kit and RNase-Free DNase I enzyme (Qiagen Inc., Valencia, CA). Using the First Strand cDNA Synthesis Kit with the Oligo-p $(\mathrm{dT})_{15}$ primer (Roche Diagnostics Corporation, Indianapolis, IN) and the thermocycler settings $\left(25^{\circ} \mathrm{C}\right.$ for $10 \mathrm{~min}$, $42^{\circ} \mathrm{C}$ for $60 \mathrm{~min}, 99^{\circ} \mathrm{C}$ for $5 \mathrm{~min}$, and $4{ }^{\circ} \mathrm{C}$ for $5 \mathrm{~min}$ ), the first 
strand cDNA synthesis was performed. To determine the RPB2 gene structure, seven primer sets, $\mathrm{m} 1 \mathrm{~A} 1 / \mathrm{m} 1 \mathrm{~B}, \mathrm{~m} 1 \mathrm{~A} 3 / \mathrm{m} 2 \mathrm{~B}$, $\mathrm{m} 2 \mathrm{~A} / \mathrm{m} 3 \mathrm{~B}, \mathrm{~m} 3 \mathrm{~A} / \mathrm{m} 4 \mathrm{~B}, \mathrm{~m} 4 \mathrm{~A} / \mathrm{m} 5 \mathrm{~B}, \mathrm{~m} 5 \mathrm{~A} / \mathrm{m} 6 \mathrm{~B}$ and $\mathrm{m} 6 \mathrm{~A} / \mathrm{m} 8 \mathrm{~B}$, were used to amplify the cDNA with the Advantage cDNA PCR Kit (BD Biosciences-Clontech, Mountain View, CA; Table 1). These primer sets provide the PCR fragments that represent the transcribed RPB2 gene-coding region. Reaction parameters were: $94^{\circ} \mathrm{C}$ for $1 \mathrm{~min}, 34$ cycles of $94^{\circ} \mathrm{C}$ for $30 \mathrm{~s}$, $55^{\circ} \mathrm{C}$ for $3 \mathrm{~min}$ and $68^{\circ} \mathrm{C}$ for $3 \mathrm{~min}$. The fragments were isolated, directly sequenced and compared with the RPB2 genomic sequence (Ueng et al. 2003b).

Seven primer sets designed from the RPB2 gene sequence of PN-w isolate Sn37-1 were used for PCR amplification of the same gene from ten PN-b, ten Paa, fourteen Pat1, two Pat2 from wild barley (foxtail barley, Hordeum jubatum;), one Pat3 from state of Washington, two Phaeosphaeria spp. from Polish ryes, one from dallis grass (Paspalum dilatatum) and sixteen other $\mathrm{PN}-\mathrm{w}$ (Tables 1-2). Two oligonucleotide primers, $\mathrm{m} 52 \mathrm{~A}$ and $\mathrm{m} 52 \mathrm{~B}$, were designed from the known sequences of PN-b, Paa and Pat1 for amplifying the corresponding PCR fragments in these Phaeosphaeria species (Table 1). These primer sets produced overlapping fragments that together represented the full-length RPB2 gene coding region. Protocols for PCR amplification were as those for the partial RPB2 gene. Isolation and direct sequencing of PCR products were conducted (Ueng et al. 2003b).

Based on the nucleotide and deduced polypeptide sequences of the RPB2 gene, phylogenetic relationships within two cereal Phaeosphaeria species were analysed using the Phylogeny Inference Package (PHYLIP) Version 3.6 (alpha2) (http:// evolution.genetics.washington.edu/phylip.html) (Felsenstein 1989). The nucleotide and polypeptide sequences were aligned with CLUSTAL_X (1.83) in a multiple sequence alignment mode (Thompson et al. 1997). From the aligned sequences, $1 \mathrm{~K}$ data sets were generated by BS re-sampling in the 'seqboot' program. The bootstrapped data sets were evaluated by the ML method with 'dnaml' and 'proml' programs. Finally, the 'consense' program was used to construct a 'tree'.

\section{Results}

Using PCR amplification with degenerate primer sets and the 'step-down' technique, a 5586 bp sequence (accession no.

Table 1 - Oligonucleotide primers used to amplify PCR products from the gene encoding the second largest protein subunit of RNA polymerase II (RPB2)

\begin{tabular}{|c|c|c|}
\hline Name & Nucleotide position (no.) ${ }^{\mathrm{a}}$ & Sequence $5^{\prime} \rightarrow 3^{\prime c}$ \\
\hline \multicolumn{3}{|c|}{ Genomic DNA fragments amplified from wheat biotype Phaeosphaeria nodorum isolate Sn37-1 } \\
\hline$f R P B 2-5 F / f R P B 2-7 c R$ & $2444-2463 / 3639-3620$ & GAYGAYMGWGATCAYTTYGG/CCCATRGCTTGYTTRCCCAT \\
\hline fRPB2-7cF/RPB2-11bR & $3620-3639 / 4713-4694$ & ATGGGYAARCAAGCYATGGG/CAATCWCGYTCCATYTCWCC \\
\hline $2 \mathrm{~A} / 16 \mathrm{~A}$ & $3251-3273 / 4346-4323$ & GAGCAGTTGGATAGCGACACTCG/GAGGGTTGATGACCAAATCAGGAG \\
\hline RPB2-3bF/21B & $1838-1860 / 2827-2806$ & GGWGGWTAYTTYATYATYAATGG/GCAAACAAGACCCCAATGCGAG \\
\hline 21B/PP1 & $2827-2806 / 5^{\prime}$ end & GCAAACAAGACCCCAATGCGAG/GTAATACGACTCACTATAGGGC \\
\hline $15 B / P P 2^{b}$ & $2788-2769 / 5^{\prime}$ end & CTTCGCCAGCTTACCGTCAC/ACTATAGGGCACGCGTGGT \\
\hline 12A/PP1 & $3568-3586 / 3^{\prime}$ end & CATTCCGTTCCCTGACCAC/GTAATACGACTCACTATAGGGC \\
\hline 22B/PP2 & $4270-4292 / 3^{\prime}$ end & CATTGGTATCACATACCGGCAAG/ACTATAGGGCACGCGTGGT \\
\hline 4B/PP1 & $4039-4061 / 3^{\prime}$ end & CGGAAAGACGGCGCCAATAGCAG/GTAATACGACTCACTATAGGGC \\
\hline 13B/PP2 & $4370-4393 / 3^{\prime}$ end & ATTGCCCATTTGGTCGAGTGTCTT/GTAATACGACTCACTATAGGGC \\
\hline \multicolumn{3}{|c|}{ Fragments amplified from total RNA of wheat biotype Phaeosphaeria nodorum isolate Sn37-1 } \\
\hline $\mathrm{m} 1 \mathrm{~A} 1 / \mathrm{m} 1 \mathrm{~B}$ & $1145-1167 / 1703-1682$ & CTCGCСTATCССТСССТСTССTT/CСTCGCGСTCCСATTTGATCTG \\
\hline $\mathrm{m} 1 \mathrm{~A} 3 / \mathrm{m} 2 \mathrm{~B}$ & $1595-1617 / 2005-1983$ & CGTATAATGGTTGCGAAAGAGAC/GATGTTGAAAGACGAGATCAGAC \\
\hline $\mathrm{m} 2 \mathrm{~A} / \mathrm{m} 3 \mathrm{~B}$ & $1983-2005 / 2629-2608$ & GTCTGATCTCGTCTTTCAACATC/CAGGCCATTTGTGATGATGCTG \\
\hline $\mathrm{m} 3 \mathrm{~A} / \mathrm{m} 4 \mathrm{~B}$ & $2608-2629 / 3193-3172$ & CAGCATCATCACAAATGGCCTG/GGTAGGATTGTTCTCTACTACG \\
\hline $\mathrm{m} 4 \mathrm{~A} / \mathrm{m} 5 \mathrm{~B}$ & $3172-3193 / 3962-3943$ & CGTAGTAGAGAACAATCCTACC/CCTTCAGTCTTAGAGTGTCG \\
\hline $\mathrm{m} 5 \mathrm{~A} / \mathrm{m} 6 \mathrm{~B}$ & $3943-3962 / 4727-4708$ & CGACACTCTAAGACTGAAGG/CGTGCGCAATCATACAATCG \\
\hline $\mathrm{m} 6 \mathrm{~A} / \mathrm{m} 8 \mathrm{~B}$ & $4708-4727 / 5078-5056$ & CGATTGTATGATTGCGCACG/GATTATCGAGAATGTGCTACTTG \\
\hline \multicolumn{3}{|c|}{ Genomic DNA fragments amplified from cereal Phaeosphaeria species } \\
\hline $\mathrm{M} 12-2 / 1 \mathrm{Bm}$ & $1103-1125 / 1709-1687$ & CAGACAAGGAGCTCATCCTCCTC/GTACATCCTCGCGCTCССАTTTG \\
\hline SM2/5B1 & $1588-1610 / 2467-2444$ & CTCGAATCGTATAATGGTTGCGA/CTTTCCGAAATGATCTCGGTCGTC \\
\hline $\mathrm{m} 2 \mathrm{~A} / \mathrm{m} 3 \mathrm{~B}$ & $1983-2005 / 2629-2608$ & GTCTGATCTCGTCTTTCAACATC/CAGGCCATTTGTGATGATGCTG \\
\hline $5 \mathrm{~A} 1 / 7 \mathrm{~B} 1$ & $2444-2467 / 3641-3619$ & GACGACCGAGATCATTTCGGAAAG/CACCCATGGCTTGCTTACCCATG \\
\hline \multirow[t]{2}{*}{$\mathrm{m} 31 \mathrm{~A} / \mathrm{m} 5 \mathrm{~B}(\mathrm{~m} 31 \mathrm{~A} / \mathrm{m} 52 \mathrm{~B})^{\mathrm{d}}$} & 2610-2629/3962-3943 (2610-2629/ & GCATCATCACAAATGGCCTG/CCTTCAGTCTTAGAGTGTCG (GCATCA \\
\hline & 3962-3943) & TCACAAATGGCCTG/CCTTCAATCGTAGAGTGTCG) \\
\hline 7A1/51 & $3619-3641 / 4617-4592$ & CATGGGTAAGCAAGCCATGGGTG/GCGTGGATCTTGTCGTCGACCATATG \\
\hline \multirow[t]{2}{*}{$\mathrm{m} 5 \mathrm{~A} / \mathrm{m} 8 \mathrm{~B}(\mathrm{~m} 52 \mathrm{~A} / \mathrm{m} 10 \mathrm{~B})^{\mathrm{d}}$} & 3943-3962/5078-5056 (3943-3962/ & CGACACTCTAAGACTGAAGG/GATTATCGAGAATGTGCTACTTG (CGAC \\
\hline & $5039-5017)$ & ACTCTACGATTGAAGG/CTAGTCGCGCACGCTGAGGCCAG) \\
\hline \multicolumn{3}{|c|}{$\begin{array}{l}\text { a Nucleotide positions are relative to the RNA polymerase II (RPB2) gene sequence of wheat biotype Phaeosphaeria nodorum isolate Sn37-1 } \\
\text { (accession no. DQ278491). } \\
\text { b PP1 and PP2 are used to amplify the ligated adaptors } 1 \text { and } 2 \text { sequences (Zhang \& Gurr 2002). } \\
\text { c The incompletely specified bases (IUB) group codes are } \mathrm{Y}=\mathrm{T} / \mathrm{C} ; \mathrm{M}=\mathrm{A} / \mathrm{C} ; \mathrm{W}=\mathrm{T} / \mathrm{A} ; \mathrm{R}=\mathrm{A} / \mathrm{G} ; \mathrm{N}=\mathrm{G} / \mathrm{A} / \mathrm{T} / \mathrm{C} \text {. } \\
\text { d The primer sets in parenthesis are used for DNA amplification of barley biotype P. nodorum, P. a. f.sp. avenaria (Paa) and P. a. f. sp. triticea (Pat1). }\end{array}$} \\
\hline
\end{tabular}


Table 2 - Isolates of Phaeosphaeria species used for analysis of the gene encoding the second largest protein subunit of RNA polymerase II (RPB2)

\begin{tabular}{|c|c|c|c|c|}
\hline Species & Original host & Year & Geographic location & GenBank accession number \\
\hline \multicolumn{5}{|c|}{ Phaeosphaeria nodorum (wheat biotype; PN-w) } \\
\hline Sn37-1 & Wheat & - & Szelejewo, Poland & DQ278491 \\
\hline 9074 & Wheat (Triticum aestivum) & 1983 & Gallatin County, MT, USA & $(=\mathrm{DQ} 278491)$ \\
\hline 9076 & Wheat & 1986 & Richland County, MT, USA & $(=\mathrm{DQ} 278491)$ \\
\hline 8408 & Wheat & 1986 & Mandan, ND, USA & $(=\mathrm{DQ} 278491)$ \\
\hline S-81-W15 & Wheat & 1981 & Sheridan, OR, USA & $(=\mathrm{DQ} 278491)$ \\
\hline 9506 & Wheat & 1987 & Mandan, ND, USA & DQ499806 \\
\hline S-87-2 & Wheat ('Oasis') & 1987 & Griffin, GA, USA & $(=\mathrm{DQ} 499806)$ \\
\hline Sn26-1 & Wheat & - & Rzeszów, Poland & DQ499807 \\
\hline $98-12981$ & Rye (Secale cereale) & 1998 & Mandan, ND, USA & $(=\mathrm{DQ} 499807)$ \\
\hline S-78-13 & Wheat & 1978 & Toluca, Mexico & $(=\mathrm{DQ} 499807)$ \\
\hline S-80-301 & Triticale (xTriticosecale) & 1980 & Williamson, GA, USA & $(=\mathrm{DQ} 499807)$ \\
\hline S-81-W12 & Wheat & 1981 & Marion County, OR & $(=\mathrm{DQ} 499807)$ \\
\hline S-81-W13 & Wheat & 1981 & Marion County, OR & $(=\mathrm{DQ} 499807)$ \\
\hline S-81-W16 & Wheat & 1981 & Harrisburg, OR, USA & $(=\mathrm{DQ} 499807)$ \\
\hline S-74-20A (ATCC200806) & Wheat & 1975 & Griffin, GA, USA & DQ499808 \\
\hline Sn27-1 & Wheat & - & Sieradz, Poland & DQ499809 \\
\hline S-81-B13B & Barley (Hordeum vulgare) & 1981 & Bledsoe, GA, USA & $(=\mathrm{DQ} 499809)$ \\
\hline \multicolumn{5}{|c|}{ Phaeosphaeria sp. (from Poland) } \\
\hline Sn23-1 & Winter rye & - & Bydgoszcz, Poland & DQ499804 \\
\hline Sn $48-1$ & Winter rye & 1995 & Jelenia Góra, Poland & DQ499805 \\
\hline \multicolumn{5}{|c|}{ Phaeosphaeria nodorum (barley biotype; PN-b) } \\
\hline$S-80-603$ & Barley & 1980 & Williamson, GA, USA & DQ499803 \\
\hline S-80-611 & Barley & 1980 & Laurinburg, NC, USA & $(=\mathrm{DQ} 499803)$ \\
\hline S-81-B9 & Barley & 1981 & Clayton, GA, USA & $(=\mathrm{DQ} 499803)$ \\
\hline S-82-13 (ATCC200805) & Barley & 1982 & Senoia, GA, USA & $(=\mathrm{DQ} 499803)$ \\
\hline S-83-2 (ATCC200841) & Barley & 1983 & Tifton, GA, USA & $(=\mathrm{DQ} 499803)$ \\
\hline S-83-7 & Barley & 1983 & Holland, VA, USA & $(=\mathrm{DQ} 499803)$ \\
\hline S-84-2 & Barley & 1984 & Moultrie, GA, USA & $(=\mathrm{DQ} 499803)$ \\
\hline S-92-7 & Barley & 1992 & Raleigh, NC, USA & $(=\mathrm{DQ} 499803)$ \\
\hline S-93-38 & Barley & 1993 & Floyd County, GA, USA & $(=\mathrm{DQ} 499803)$ \\
\hline S-93-39 & Barley & 1993 & Pike County, GA, USA & $(=\mathrm{DQ} 499803)$ \\
\hline \multicolumn{5}{|c|}{ Phaeosphaeria avenaria f. sp. avenaria (Paa) } \\
\hline ATCC12277 & Oat (Avena sativa) & - & USA & DQ499810 \\
\hline Sat002NY-84 & Wheat & 1984 & New York, USA & DQ499811 \\
\hline 1920WRS & Oat & 2002 & Manitoba, Canada & DQ499812 \\
\hline Saa001NY-85 & Oat & 1985 & New York, USA & DQ499813 \\
\hline 1919WRS & Oat & 2002 & Manitoba, Canada & $(=\mathrm{DQ} 499813)$ \\
\hline ATCC58582 & Wheat & 1984 & New York, USA & DQ499814 \\
\hline ATCC58583 & Wheat & 1984 & New York, USA & $(=\mathrm{DQ} 499814)$ \\
\hline 5413 & Oat & 1983 & Ontario, Canada & DQ499815 \\
\hline 1921WRS & Oat & 2002 & Manitoba, Canada & $(=\mathrm{DQ} 499815)$ \\
\hline Sa37-2 & Oat & 2001 & Radzików, Poland & DQ499816 \\
\hline \multicolumn{5}{|c|}{ Phaeosphaeria avenaria f. sp. triticea (Pat1) } \\
\hline Sat24-1 & Wheat & - & Warmińsko-Mazurskie, Poland & DQ499801 \\
\hline $10052-2$ & Wheat & 1988 & Langdon, ND, USA & $(=\mathrm{DQ} 499801)$ \\
\hline 12618 & Wheat & 1995 & Dickinson, ND, USA & $(=\mathrm{DQ} 499801)$ \\
\hline 12889 & Wheat & 1997 & Mandan, ND, USA & $(=\mathrm{DQ} 499801)$ \\
\hline $13050-2$ & Barley & 1998 & Dunn County, ND, USA & $(=\mathrm{DQ} 499801)$ \\
\hline 13061 & Barley & 1998 & Morton County, ND, USA & $(=\mathrm{DQ} 499801)$ \\
\hline $13077-2$ & Barley & 1998 & Towner County, ND, USA & $(=\mathrm{DQ} 499801)$ \\
\hline Sa38-1 & Oat & 2001 & Radzików, Poland & $(=\mathrm{DQ} 499801)$ \\
\hline Sa39-2 & Oat & 2001 & Radzików, Poland & $(=\mathrm{DQ} 499801)$ \\
\hline Sat22-2 & Rye & 1995 & Podkarpackie, Poland & $(=\mathrm{DQ} 499801)$ \\
\hline Sat23-2 & Triticale & 1995 & Mazowieckie, Poland & $(=\mathrm{DQ} 499801)$ \\
\hline Sat23-8 & Triticale & 1995 & Mazowieckie, Poland & $(=\mathrm{DQ} 499801)$ \\
\hline ATCC26374 & Foxtail barley (Hordeum jubatum) & 1972 & Minnesota, USA & DQ499802 \\
\hline ATCC26375 & Foxtail barley & 1972 & Minnesota, USA & $(=\mathrm{DQ} 499802)$ \\
\hline \multicolumn{5}{|c|}{ Phaeosphaeria avenaria f. sp. triticea (Pat2) } \\
\hline ATCC26370 & Foxtail barley & 1972 & Minnesota, USA & DQ499800 \\
\hline ATCC26377 & Foxtail barley & 1972 & Minnesota, USA & $(=\mathrm{DQ} 499800)$ \\
\hline \multicolumn{5}{|c|}{ Phaeosphaeria avenaria f. sp. triticea (Pat3) } \\
\hline S-81-W10 & Wheat & 1981 & Washington, USA & DQ499799 \\
\hline \multicolumn{5}{|l|}{ Phaeosphaeria sp. } \\
\hline S-93-48 & Dallis grass (Paspalum dilatatum) & 1993 & Griffin, GA, USA & DQ499798 \\
\hline
\end{tabular}


DQ278491) containing the RPB2 gene was obtained from the $\mathrm{PN}-\mathrm{w}$ isolate Sn37-1 (Fig 2). Three programs were used to delineate the RPB2 gene and its associated signals. Based on the Promoter Scan analysis (http://bimas.dcrt.nih.gov), the transcription start site (TSS) of the RPB2 gene was detected at nt758. Additionally, several specific protein factor binding sequences required for the regulation of RPB2 gene transcription were found in the upstream promoter region of the RPB2

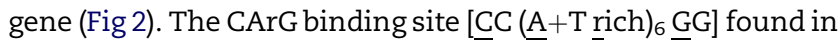
the promoter regions of human and mouse cardiac $\alpha$-actin genes was present at nt728-nt737 (Miwa et al. 1987). Two activating transcription factor (ATF) sites, (G/T)(A/T)CGTCA, identical to several distinct nucleoprotein-binding sites in the early region $1 \mathrm{~A}$ (E1A)-inducible adenovirus E3 promoter, were detected at nt261-nt267 and nt698-nt704 (Hurst \& Jones 1987). Other sequences similar to the binding sites for yeast (Saccharomyces cerevisiae) autonomously replicating sequence-binding factor 1 (ABF1; ATCAN $_{6}$ ACGA), general regulatory factor I (GRFI; RMACCCANNCAYY) and ribosomal protein genes box (RPG; ACACCCANNCA), were also found at nt115-nt128, nt550-nt562 and nt625-nt635, respectively (Fig 2) (Buchman et al. 1988). An analysis by Hamming-Clustering Methods for eukaryotic genes (HCtata and HCpolya programs) (http://www.itb.cnr.it/sun/webgene/) showed that the TATA signal was at nt731 and the Poly-A tailing signal was at nt5202. By using the FGENESH program (http://www.softberry. com) with Aspergillus as the organism parameter, two exons (nt1199-nt4821 and nt4874-nt5039) and one intron (nt4822- nt4873) were predicted. The intron position of the RPB2 gene in $\mathrm{PN}-\mathrm{w}$ was experimentally confirmed by cDNA sequencings.

The combined $3789 \mathrm{bp}$ nucleotide sequence of two exons of the RPB2 gene encoded a 1262 amino acid polypeptide (Fig 2). Like the $\mathrm{C}$-terminal structures of the $\beta$ subunit in prokaryotes and the RPB2 polypeptide in yeast RNA polymerase II, many conserved structural features were present in the C-terminal of RPB2 polypeptide in $\mathrm{PN}-\mathrm{w}$ (Fig 2). They included a putative consensus zinc-binding motif $\left(\mathrm{CX}_{2} \mathrm{CX}_{15} \mathrm{CX}_{2} \mathrm{C}\right)$ (aa1198aa1220), a purine nucleotide-binding motif (GX $\mathrm{GKKT}_{5}$ ) (aa942aa950), two amino acid sequence clusters (aa997-aa1014 and aa1129-aa1137) involved in the formation of the active site of RNA polymerase II, which contained two nucleotide-binding lysine (K) (aa1014 and aa1137) (James et al. 1991; Treich et al. 1991; Sweetser et al. 1987; Grachev et al. 1989). As in other eukaryotic RPB2 polypeptides, there was a conserved prokaryotic RNase-like domain in the P. nodorum peptide (aa323aa426) (Fig 2) (Shirai \& Gō 1991). There were 531 amino acids (42\% identity) in the full-length RPB2 polypeptides that are conserved in 11 ascomycetes (Fig 2).

The RPB2 gene coding sequence amplified from cereal Phaeosphaeria species differed in length (Table 3). The RPB2 gene from PN-w, homothallic Pat1, heterothallic Pat2, and Phaeosphaeria sp. from Polish ryes had the same length (3841 bp), while the same gene from PN-b, Paa, Pat3 and Phaeosphaeria sp. from dallis grass was $3840 \mathrm{bp}$ in size. The size difference was due to variations in intron size (Table 3). Nucleotide substitutions were not found in ten PN-b isolates

Table 3 - Structure of RNA polymerase (RPB2) gene in cereal Phaeosphaeria species

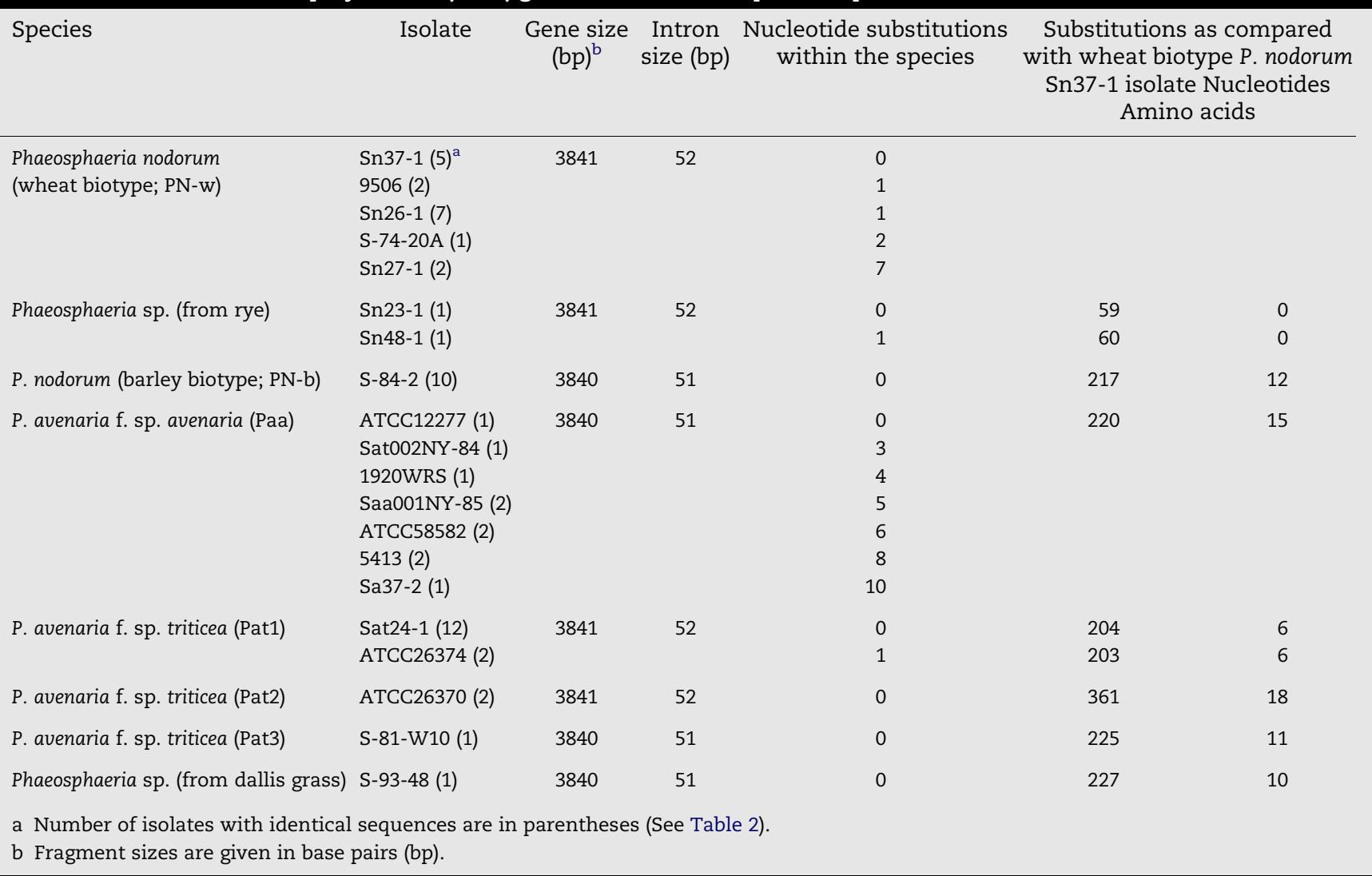


and 12 homothallic Pat1 isolates from commercially cultivated cereals. However, one nucleotide substitution (nt3650) in the intron was found in two Pat1 isolates from wild barley. Nucleotide sequence diversity of the RPB2 gene within the species was much less than inter-species. From one to ten substitutions were found in $\mathrm{PN}-\mathrm{w}$, Phaeosphaeria sp. from Polish ryes, and Paa (Table 3). In comparison with $\mathrm{PN}-\mathrm{w}$ isolate Sn37-1, sequence differences in other Phaeosphaeria isolates were 59 to $361 \mathrm{bp}$ (Table 3).

Many of the nucleotide changes occurred in either intron or the third position of amino acid coding triplets and did not affect the amino acid composition in RPB2 polypeptides. There were five nucleotide changes in the intron and 54-55 changes in exons in the RPB2 gene between PN-w isolate Sn37-1 and two Phaeosphaeria sp. from Polish ryes, but no amino acid changes were found in their RPB2 polypeptides (Table 3). There were six to 18 substitutions in the deduced amino acid sequences of RPB2 polypeptides of other Phaeosphaeria species as compared with PN-w (Table 3). It appears that the RPB2 polypeptide in two heterothallic Pat2 isolates from wild barley is the most diversified among the cereal Phaeosphaeria species (Table 3). All amino acid divergences were present in two regions of the RPB2 polypeptide, which were from aa3 to aa299 and from aa572 to aa802. Two other amino acid substitutes (V $\leftrightarrow$ I), which occurred at aa345 and aa1046, were found in two heterothallic Pat2 isolates. The structural features conserved in the C-terminal and the prokaryotic RNase-like domains in the $\mathrm{N}$-terminal were not affected.

The phylogenetic relationships based on the deduced RPB2 amino acid sequence was the same as that based on the RPB2 gene nucleotide sequence in Phaeosphaeria species (Fig 3). It appears that homothallic Pat1, heterothallic Pat2, PN-w and Phaeosphaeria sp. from rye are closely related, and PN-b, Paa, Pat3 and Phaeosphaeria sp. from dallis grass form a single clade (Fig 3).

\section{Discussion}

The phylogenetic positions of organisms at the primary kingdoms of life might be misplaced due to the usage of genes with

A

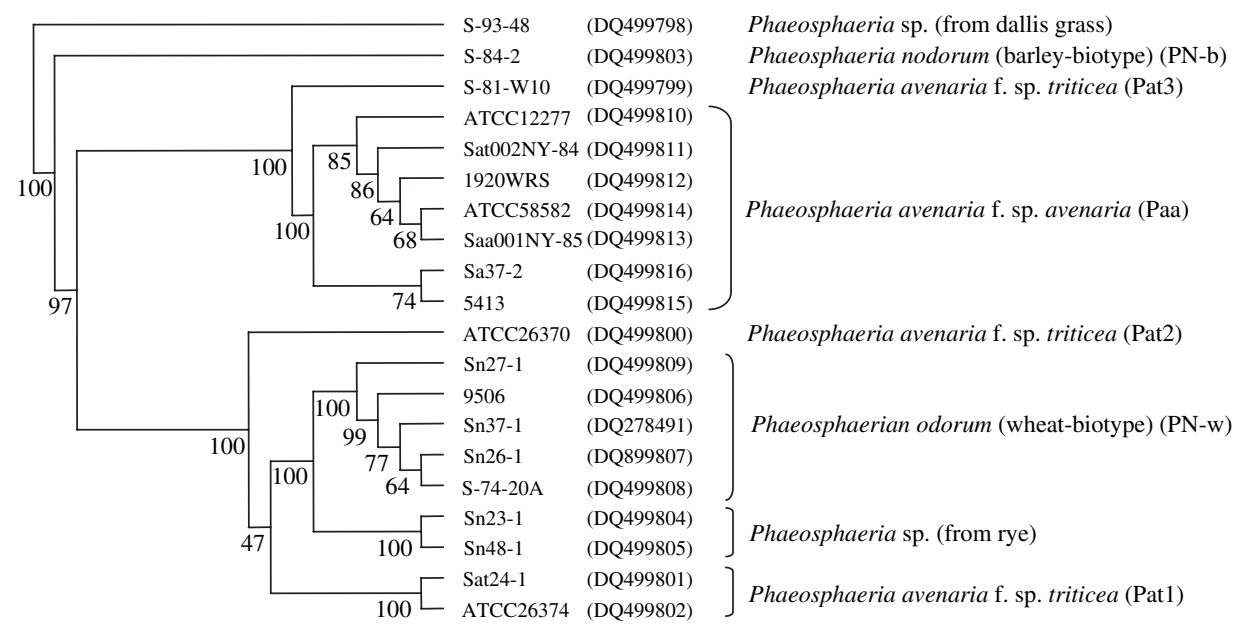

B

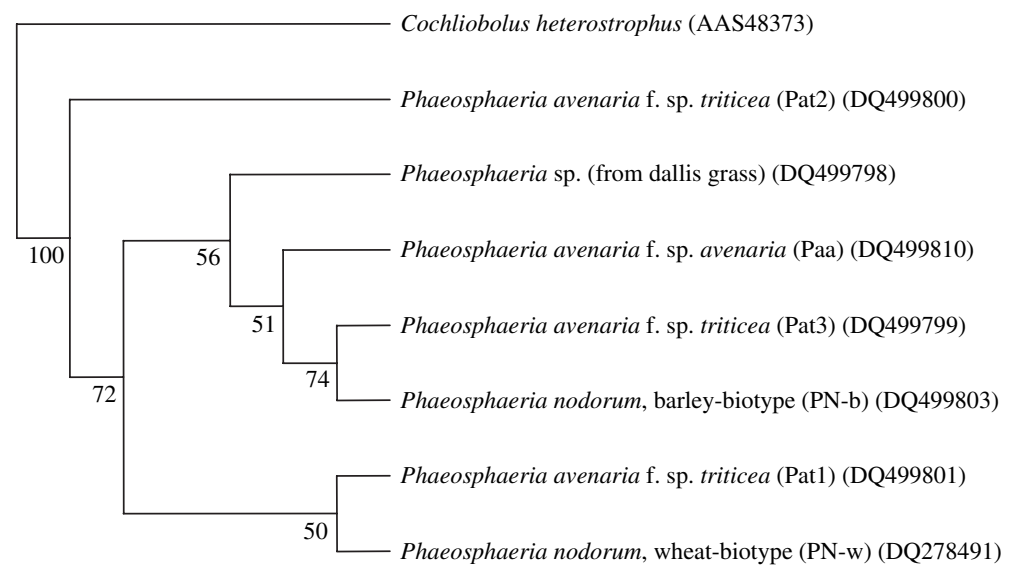

Fig 3 - Phylogenetic relationship based on the full-length nucleotide (A) and its deduced amino acid (B) sequences of the second largest subunit of RNA polymerase II (RPB2) gene in cereal Phaeosphaeria species. GenBank accession numbers are given in parentheses. The maize pathogen, Cochliobolus heterostrophus, was used as an out-group in B. BS values (with $1 \mathrm{~K}$ replications) of the internal branches are indicated. The amino acid sequences of the RPB2 gene in PN-w and Phaeosphaeria sp. from rye are identical. 
an unusually high rate of sequence divergence for analysis. The RPB2 gene encodes a protein with a modest rate of evolutionary change, and its polypeptide sequence has been used for phylogenetic studies in green plants (Denton et al. 1998), archaebacteria (Iwabe et al. 1991), red algae (Stiller \& Hall 1997), and ascomycetes, including lichen-forming taxa (Liu et al. 1999; Liu \& Hall 2004; Reeb et al. 2004). The sequence diversities in the RPB2 gene also provide useful phylogenetic relationships at inter- and intra-specific levels in the genera Trichoderma/Hypocrea and Leotia (Chaverri et al. 2003; Zhong \& Pfister 2004).

In order to understand the phylogenetic relationships of PN-w with other Loculoascomycetes, ten other full-length RPB2 polypeptide sequences (1208-1287aa in length) of ascomycetes deposited in the GenBank were used for phylogenetic analysis. It appears that Cochliobolus heterostrophus and $\mathrm{PN}-\mathrm{w}$ are more closely related to each other than to the other ascomycetes (Fig 4). By using the FGENESH program with Aspergillus as the organism parameter, the 3852 bp RPB2 gene coding sequence (nt794-nt4645) in C. heterostrophus (accession no. AY533025) contains a $63 \mathrm{bp}$ intron and $3789 \mathrm{bp}$ nucleotide sequence in two exons, which encoded a protein of 1262 amino acid residues. The RPB2 polypeptides in both $C$. heterostrophus and $\mathrm{PN}-\mathrm{w}$ were the same length and had a $90.3 \%$ amino acid identity (Ueng, unpublished data). The identities of RPB2 polypeptides between $\mathrm{PN}-\mathrm{w}$ and the other nine ascomycetes listed in Fig 4 were as low as 63.6-66.9\%. In previous studies, high identities between $\mathrm{C}$. heterostrophus and $\mathrm{PN}-\mathrm{w}$ were reported in the mating-type gene-encoded proteins and glyceraldehyde-3-phosphate dehydrogenase (GPD) (EC1.2.1.12) enzyme (Bennett et al. 2003; Ueng et al. 2003b). The close relationship between these two organisms was re-confirmed with RPB2 polypeptide sequence in this study (Fig 4).

A P. nodorum gene encoding a hypothetical protein similar to DNA-dependent RNA polymerase II RPB140 of Curvularia brachyspora (accession no. AAF19075) was recently reported (www.broad.mit.edu/annotation/genome; SNOG_11456.1). The
3842 bp coding sequence was predicted to have three introns (42, 193 and $100 \mathrm{bp}$ in size) and four exons, and encoded an 1168 amino acid polypeptide. By using the FGENESH program with Aspergillus as the organism parameter, this 3842 bp coding sequence (SNOG_11456.1) was predicted to have two introns (130 and $52 \mathrm{bp}$ in size) and three exons, and encoded a 1216-amino acid polypeptide. As compared with the RPB2 gene genomic sequence in PN-w Sn37-1 isolate (accession no. DQ278491), there was one nucleotide substitution at nt876 (from ' $C$ ' to ' $T$ ') and one extra ' $T$ ' nucleotide between nt1188 and nt1189 in this gene coding sequence (SNOG_11456.1). As the intron position and the coding sequence size of the RPB2 gene were confirmed experimentally by genomic and cDNA sequencing in this study, the prediction that it contains one intron using the FGENESH program appears to be correct. The presence of an extra ' $\mathrm{T}$ ' nucleotide in the P. nodorum gene (SNOG_11456.1) results in the prediction of a different gene structure using the FGENESH program.

The intron position within the RPB2 gene may reflect its gain and/or loss during fungal evolution, but it might not be reliable as a phylogenetic character (Liu et al. 1999). However, the intron position of the RPB2 gene in $\mathrm{PN}-\mathrm{w}$ is the same as in three other high ascomycetes. The intron is inserted between the second and third codon positions of alanine (A) in aa1202 of Aspergillus fumigatus (accession no. EAL84702), aa1208 of Cochliobolus heterostrophus (accession no. AAS48373), aa1234 of Neurospora crassa (accession no. CAD70445) and aa1208 of $\mathrm{PN}-\mathrm{w}$ (accession no. DQ278491). The only lower ascomycete reported to have an intron in the RPB2 gene is fission yeast, Schizosaccharomyces pombe (accession no. BAA02600). The intron is inserted between the first and second codon positions of aa6 tyrosine $(\mathrm{Y})$.

Stagonospora blotch diseases of cereals are a disease complex caused by a number of fungi that are not always closely related (Scharen \& Sanderson 1985; Sprague 1950). Application of molecular approaches may complement the morphologically based classification and facilitate the identification of

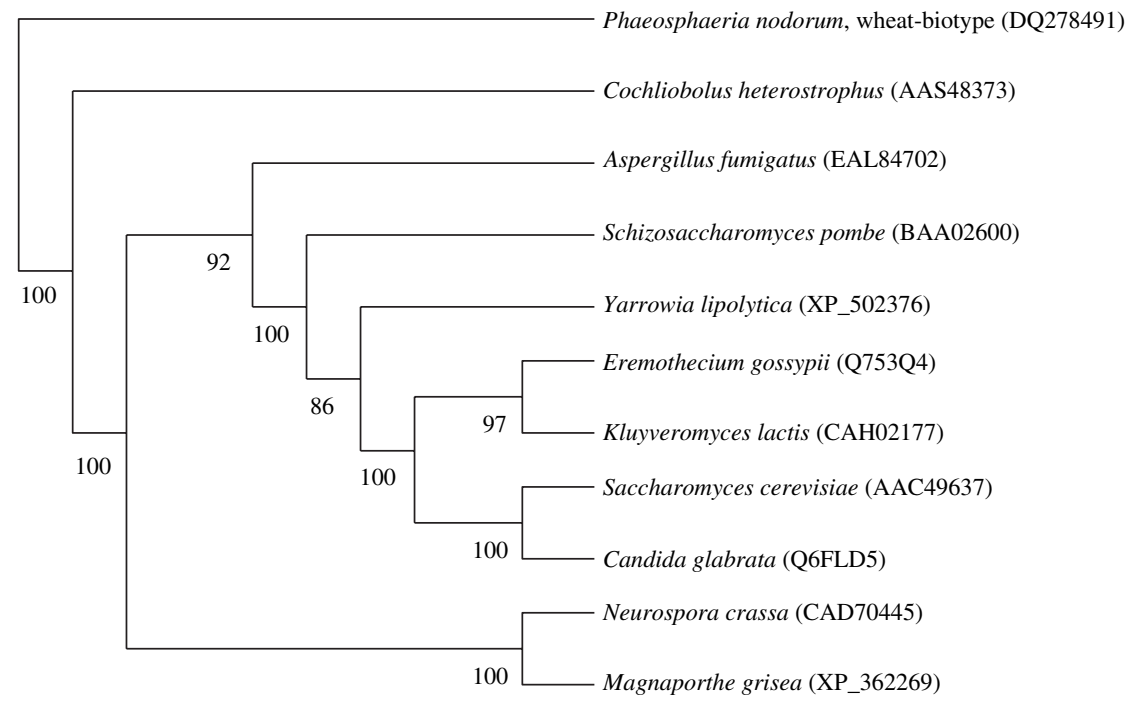

Fig 4 - Phylogenetic relationship based on the deduced polypeptide sequences of the second largest subunit of RNA polymerase II (RPB2) in ascomycetes. GenBank accession numbers are given in parentheses. BS values (with $1 \mathrm{~K}$ replications) of the internal branches are indicated. 
Table 4 - Comparison of endonuclease restriction of PCR-amplified RNA polymerase (RPB2) gene products in Phaeosphaeria leaf blotch pathogens

\begin{tabular}{|c|c|c|c|c|c|c|c|c|c|}
\hline \multirow{2}{*}{$\begin{array}{l}\text { Endonulcease } \\
\text { enzyme }\end{array}$} & \multirow{2}{*}{$\begin{array}{l}\text { Restriction } \\
\text { site (no.) }\end{array}$} & \multirow{2}{*}{$\begin{array}{c}\text { PN-w } \\
\text { (332 bp) }\end{array}$} & \multirow{2}{*}{$\begin{array}{l}\text { PN-rye } \\
\text { (332 bp) }\end{array}$} & \multirow{2}{*}{$\begin{array}{c}\text { PN-b } \\
\text { (331 bp) }\end{array}$} & \multirow{2}{*}{$\begin{array}{c}\text { Paa } \\
\text { (331 bp) }\end{array}$} & \multicolumn{3}{|c|}{ Pat } & \multirow{2}{*}{$\begin{array}{l}\text { P-93-48 } \\
\text { (331 bp) }\end{array}$} \\
\hline & & & & & & Pat1 (332 bp) & Pat2 (332 bp) & Pat3 331 bp) & \\
\hline AvaII & 301 (302) & - & 30,302 & 30,301 & 30,301 & 30,302 & - & 30,301 & 30,301 \\
\hline BanII & 107 & - & - & - & - & - & 107,225 & - & - \\
\hline BglII & 164 & - & - & - & - & - & - & - & 164,167 \\
\hline DpnI & 167 & - & - & - & - & 165,167 & - & - & - \\
\hline HaeII & 46 & - & - & 46,285 & 46,285 & 46,286 & 46,286 & 46,285 & 46,285 \\
\hline HinfI & 103 (104) & 103,229 & 103,229 & 103,228 & 104,227 & - & - & 103,228 & 103,228 \\
\hline SspI & 280 & - & - & - & 51,280 & - & - & - & - \\
\hline TaqI & $140(145)$ & 140, 192 & 140, 192 & - & - & - & 145,187 & - & - \\
\hline XbaI & 161 & - & - & - & 161,170 & - & - & 161,170 & - \\
\hline
\end{tabular}

An $\mathrm{m} 6 \mathrm{~A} / \mathrm{m} 10 \mathrm{~B}$ primer set was used to amplify the 331-332 bp fragments from wheat biotype Phaeosphaeria nodorum (PN-w), isolates from Polish ryes (PN-rye), barley biotype P. nodorum (PN-b), P. avenaria f.sp. avenaria (Paa), P. avenaria f.sp. triticea (Pat) including homothallic isolates (Pat1), heterothallic isolates (Pat2) and S-81-W10 isolate from state of Washington (Pat3), and P-93-48 isolate from dallis grass. Protocols for PCR amplification were the same as those for genomic DNA gene amplification in Materials and Methods. After PCR reaction, a $4 \mu \mathrm{l}$ sample was used for enzymatic restriction following the standard procedures (Promega, Madison, Wisconsin, USA). Fragment sizes are given in base pairs (bp). -, Not cut by endonuclease enzymes.

the Stagonospora complex in cereal blotch diseases. Recently, PCR-based methods have been used to distinguish plant pathogens in a mixed infection and to detect genetic variation in a pathogen population (Fraaije et al. 2001; McCartney et al. 2003). In addition to previous studies using ITS region sequence, the mat1 gene conserved region, and the partial gpd, bgl1 and tubA genes were used to distinguish cereal and other unidentified Phaeosphaeria species (Malkus et al. 2005; Reszka et al. 2005; Ueng et al. 1998, 2003a,b). In this study, enzymatic restriction patterns of the RPB2 gene fragments amplified with the $\mathrm{m} 6 \mathrm{~A} / \mathrm{m} 10 \mathrm{~B}$ primer set are proposed (Table 4$)$. The endonuclease restriction sites were deduced from the sequencing data and were experimentally demonstrated by PCR amplification, enzymatic restriction and agarose gel electrophoresis (Ueng, unpublished data). Nucleotide substitutions occurring in the amplified fragments did not affect the enzymatic restrictions and the banding patterns in agarose electrophoresis gels (Ueng, unpublished data). It appears that specific digestions by BanII, BglII, DpnI and SspI can identify heterothallic Pat2, P-93-48 isolate from dallis grass, homothallic Pat1 and Paa, respectively. The AvaII enzymatic restriction can separate PN-w from Phaeosphaeria sp. of Polish rye. Digestion with a combination of three other enzymes (HaeII, TaqI and XbaI) may distinguish PN-b and Pat3 from other Phaeosphaeria species tested.

The polypeptide sequences encoded by the gpd and tubA genes are identical in cereal Phaeosphaeria species tested, with the exception of heterothallic Pat2 isolates from wild barley (Malkus et al. 2005; Ueng et al. 2003b). This indicates that the cereal Phaeosphaeria species are closely related phylogenetically. However, based on the nucleotide sequences, the correlation between homothallic Pat1 and PN-w are different in different genes. In the gpd, tubA and bgl1 genes, homothallic Pat1 is more closely related to the phylogenetic clade containing Paa, Pat3 and PN-b than to PN-w (Malkus et al. 2005; Reszka et al. 2005; Ueng et al. 2003b). Homothallic Pat1 apparently is closely related to $\mathrm{PN}-\mathrm{w}$, Pat2 and Phaeosphaeria sp. from rye in the RPB2 gene (Fig 3A). To further understand the phylogenetic relationships in cereal Phaeosphaeria species, more sequence data from numerous genes are required for comparison.

\section{Acknowledgements}

We thank Rosemarie Hammond of the USD-ARS and Wei of Tokyo University, Japan, for reviewing the manuscript.

\section{R E F E R E N C E S}

Archambault J, Friesen JD, 1993. Genetics of eukaryotic RNA polymerases I, II. III. Microbiological Reviews 57: 703-724.

Baldauf SL, Roger AJ, Wenk-Siefert I, Doolittle WF, 2000. A kingdom-level phylogeny of eukaryotes based on combined protein data. Science 290: 972-977.

Bennett RS, Yun SH, Lee TY, Turgeon BG, Arseniuk E, Cunfer BM, Bergstrom GC, 2003. Identity and conservation of mating type genes in geographically diverse isolates of Phaeosphaeria nodorum. Fungal Genetics and Biology 40: 25-37.

Buchman AR, Kimmerly WJ, Rine J, Kornberg RD, 1988. Two DNAbinding factors recognize specific sequences at silencers, upstream activating sequences, autonomously replicating sequences, and telomeres in Saccharomyces cerevisiae. Molecular and Cellular Biology 8: 21-225.

Chaverri P, Castlebury LA, Overton BE, Samuels GJ, 2003. Hypocrea/Trichoderma: species with conidiophore elongations and green conidia. Mycologia 95: 1100-1140.

Chaverri P, Samuels GJ, 2003. Hypocrea/Trichoderma (Ascomycota, Hypocreales, Hypocreaceae): species with green ascospores. Studies in Mycology 48: 155.

Couture L, 1989. A summary of diseases of oats in Quebec in 1988. Canadian Plant Disease Survey 69: 35.

Cunfer BM, 1997. Taxonomy and nomenclature of Septoria and Stagonospora species on small grain cereals. Plant Disease 81: 427-428.

Cunfer BM, 2000. Stagonospora and Septoria diseases of barley, oat, and rye. Canadian Journal of Plant Pathology 22: 332-348. 
Cunfer BM, Ueng PP, 1999. Taxonomy and identification of Septoria and Stagonospora species on small-grain cereals. Annual Review of Phytopathology 37: 267-284.

Denton AL, McConaughy BL, Hall BD, 1998. Usefulness of RNA polymerase II coding sequences for estimation of green plant phylogeny. Molecular Biology and Evolution 15: 1082-1085.

Felsenstein J, 1989. PHYLIP - Phylogeny Inference Package (Version 3.2). Cladistics 5: 164-166.

Fraaije BA, Lovell DJ, Coelho JM, Baldwin S, Hollomon DW, 2001. PCR-based assays to assess wheat varietal resistance to blotch (Septoria tritici and Stagonospora nodorum) and rust (Puccinia striiformis and Puccinia recondita) diseases. European Journal of Plant Pathology 107: 905-917.

Grachev MA, Lukhtanov EA, Mustaev AA, Zaychikov EF, Abdukayumov MN, Rabinov IV, Richter VI, Skoblov YS, Chistyakov PG, 1989. Studies of the functional topography of Escherichia coli RNA polymerase. A method for localization of the sites of affinity labelling. European Journal of Biochemistry 180: 577-585.

Hansen K, LoBuglio KF, Pfister DH, 2005. Evolutionary relationships of the cup-fungus genus Peziza and Pezizaceae inferred from multiple nuclear genes: RPB2, $\beta$-tubulin, and LSU rDNA. Molecular Phylogenetics and Evolution 36: 1-23.

Holmes SJI, Colhoun J, 1970. Septoria nodorum as a pathogen of barley. Transactions of the British Mycological Society 55: 321325.

Hurst HC, Jones NC, 1987. Identification of factors that interact with the E1A-inducible adenovirus E3 promoter. Genes \& Development 1: 1132-1146.

Ishihama A, Kimura M, Mitsuzawa H, 1998. Subunits of yeast RNA polymerases: structure and function. Current Opinion in Microbiology 1: 190-198.

Iwabe N, Kuma K, Kishino H, Hasegawa M, Miyata T, 1991. Evolution of RNA polymerases and branching patterns of the three major groups of archaebacteria. Journal of Molecular Evolution 32: 70-78.

James P, Whelen S, Hall BD, 1991. The RET1 gene of yeast encodes the second-largest subunit of RNA polymerase III. Journal of Biological Chemistry 266: 5616-5624.

Johnson T, 1947. A form of Leptosphaeria avenaria on wheat in Canada. Canadian Journal of Research, Section C, Botanical Science 25: 259-270.

Liu YJ, Hall BD, 2004. Body plan evolution of ascomycetes, as inferred from an RNA polymerase II phylogeny. Proceedings of the National Academy of Sciences of the USA 101: 4507-4512.

Liu YJ, Whelen S, Hall BD, 1999. Phylogenetic relationships among ascomycetes: evidence from an RNA polymerase II subunit. Molecular Biology and Evolution 16: 1799-1808.

Lumbsch HT, 2000. Phylogeny of filamentous ascomycetes. Naturwissenschaften 87: 335-342.

Lutzoni F, Kauff F, Cox CJ, McLaughlin D, Celio G, Dentinger B, Padamsee M, Hibbett D, James TY, Baloch E, Grube M, Reeb V, Hofstetter V, Schoch C, Arnold AE, Miadlikowska J, Spatafora J, Johnson D, Hambleton S, Crockett M, Shoemaker R, Sung GH, Lücking R, Lumbsch T, O'Donnell K, Binder M, Diederich P, Ertz D, Gueidan C, Hansen K, Harris RC, Hosaka K, Lim YW, Matheny B, Nishida H, Pfister D, Rogers J, Rossman A, Schmitt I, Sipman H, Stone J, Sugiyama J, Yahr R, Vilgalys R, 2004. Assembling the fungal tree of life: progress, classification, and evolution of subcellular traits. American Journal of Botany 91: 1446-1480.

Malkus A, Reszka E, Chang CJ, Arseniuk E, Chang PFL, Ueng PP, 2005. Sequence diversity of $\beta$-tubulin (tubA) gene in Phaeosphaeria nodorum and P. avenaria. FEMS Microbiology Letters 249: 49-56.

McCartney HA, Foster SJ, Fraaije BA, Ward E, 2003. Molecular diagnostics for fungal plant pathogens. Pest Management Science 59: 129-142.
Miller AN, Huhndorf SM, 2005. Multi-gene phylogenies indicate ascomal wall morphology is a better predictor of phylogenetic relationships than ascospore morphology in the Sordariales (Ascomycota, Fungi). Molecular Phylogenetics and Evolution 35: 60-75.

Miwa T, Boxer LM, Kedes L, 1987. CArG boxes in the human cardiac $\alpha$-actin gene are core binding sites for positive transacting regulatory factors. Proceedings of the National Academy of Sciences, USA 84: 6702-6706.

Noble M, Montgomerie IG, 1954. Seed-borne oat diseases. Strawbreaking and brown foot-rot. Scottish Agriculture 34: 51-52.

Reeb V, Lutzoni F, Roux C, 2004. Contribution of RPB2 to multilocus phylogenetic studies of the euascomycetes (Pezizomycotina, Fungi) with special emphasis on the lichen-forming Acarosporaceae and evolution of polyspory. Molecular Phylogenetics and Evolution 32: 1036-1060.

Reszka E, Arseniuk E, Malkus A, Chung KR, O’Neill NR, Song QJ, Ueng PP, 2006. A new biotype of Phaeosphaeria sp. of uncertain affinity causing Stagonospora leaf blotch disease in cereals in Poland. Plant Disease 90: 113.

Reszka E, Chung KR, Tekauz A, Malkus A, Arseniuk E, Krupinsky JM, Tsang H, Ueng PP, 2005. Presence of $\beta$-glucosidase (bgl1) gene in Phaeosphaeria nodorum and Phaeosphaeria avenaria f.sp. triticea. Canadian Journal of Botany 83: 1001-1014.

Richardson MJ, Noble M, 1970. Septoria species on cereals-a note to aid their identification. Plant Pathology 19: 159-163.

Sakurai H, Ishihama A, 1997. Gene organization and protein sequence of the small subunits of Schizosaccharomyces pombe RNA polymerase II. Gene 196: 165-174.

Scharen AL, Sanderson FR, 1985. Identification, distribution and nomenclature of the Septoria species that attack cereals. In: Scharen AL (ed), Septoria of Cereals. USDA-ARS, ARS-12, Washington, DC, pp. 37-41.

Shaw DE, 1957. Studies on Leptosphaeria avenaria f. sp. avenaria. Canadian Journal of Botany 35: 97-112.

Shirai T, Gō M, 1991. RNase-like domain in DNA-directed RNA polymerase II. Proceedings of the National Academy of Sciences, USA 88: 9056-9060.

Sicheritz-Pontén T, Andersson SGE, 2001. A phylogenomic approach to microbial evolution. Nucleic Acids Research 29: 545-552.

Smedegård-Petersen V, 1974. Leptosphaeria nodorum (Septoria nodorum), a new pathogen on barley in Denmark, and its physiologic specialization on barley and wheat. Friesia 10 : 251-264.

Sprague R, 1950. Diseases of Cereals and Grasses in North America. Ronald Press, New York.

Staats M, van Baarlen P, van Kan JAL, 2005. Molecular phylogeny of the plant pathogenic genus Botrytis and the evolution of host specificity. Molecular Biology and Evolution 22: 333-346.

Stiller JW, Hall BD, 1997. The origin of red algae: implications for plastid evolution. Proceedings of the National Academy of Sciences of the USA 94: 4520-4525.

Sweetser D, Nonet M, Young RA, 1987. Prokaryotic and eukaryotic RNA polymerases have homologous core subunits. Proceedings of the National Academy of Sciences, USA 84: 1192-1196.

Thompson JD, Gibson TJ, Plewniak F, Jeanmougin F, Higgins DG, 1997. The CLUSTAL_X windows interface: flexible strategies for multiple sequence alignment aided by quality analysis tools. Nucleic Acids Research 25: 4876-4882.

Treich I, Riva M, Sentenac A, 1991. Zinc-binding subunits of yeast RNA polymerases. Journal of Biological Chemistry 266: 2197121976.

Ueng PP, Bergstrom GC, Slay RM, Geiger EA, Shaner G, Scharen AL, 1992. Restriction fragment length polymorphisms in the wheat glume blotch fungus, Phaeosphaeria nodorum. Phytopathology 82: 1302-1305. 
Ueng PP, Dai Q, Cui K, Czembor PC, Cunfer BM, Tsang H, Arseniuk E, Bergstrom GC, 2003a. Sequence diversity of mating-type genes in Phaeosphaeria avenaria. Current Genetics 43: 121-130.

Ueng PP, Reszka E, Chung KR, Arseniuk E, Krupinsky JM, 2003b. Comparison of glyceraldehyde-3-phosphate dehydrogenase genes in Phaeosphaeria nodorum and P. avenaria species. Plant Pathology Bulletin 12: 255-268.

Ueng PP, Subramaniam K, Chen W, Arseniuk E, Wang L, Cheung AM, Hoffmann GM, Bergstrom GC, 1998. Intraspecific genetic variation of Stagonospora avenae and its differentiation from S. nodorum. Mycological Research 102: 607-614.

Weber GF, 1922a. Septoria diseases of cereals. I. Speckled blotch of oats caused by Leptosphaeria. Phytopathology 12: 449-470.

Weber GF, 1922b. Septoria diseases of cereals. II. Septoria diseases of wheat. Phytopathology 12: 537-585.
Young RA, 1991. RNA polymerases II. Annual Review of Biochemistry 60: 689-715.

Zhang N, Blackwell M, 2001. Molecular phylogeny of dogwood anthracnose fungus (Discula destructiva) and the Diaporthales. Mycologia 93: 355-365.

Zhang N, Blackwell M, 2002. Molecular phylogeny of Melanospora and similar pyrenomycetous fungi. Mycological Research 106: 148-155.

Zhang Z, Gurr SJ, 2002. A 'step down' PCR-based technique for walking into and the subsequent direct-sequence analysis of flanking genomic DNA. Methods in Molecular Biology 192: 343350.

Zhong Z, Pfister DH, 2004. Phylogenetic relationships among species of Leotia (Leotiales) based on ITS and RPB2 sequences. Mycological Progress 3: 237-246. 